\title{
ARTICLE
}

\section{Long-distance endosome trafficking drives fungal effector production during plant infection}

\author{
Ewa Bielska ${ }^{1, \star}$, Yujiro Higuchi ${ }^{1, \star, \dagger}$, Martin Schuster ${ }^{1, \star}$, Natascha Steinberg ${ }^{1, \dagger}$, Sreedhar Kilaru ${ }^{1}$, \\ Nicholas J. Talbot ${ }^{1} \&$ Gero Steinberg ${ }^{1}$
}

To cause plant disease, pathogenic fungi can secrete effector proteins into plant cells to suppress plant immunity and facilitate fungal infection. Most fungal pathogens infect plants using very long strand-like cells, called hyphae, that secrete effectors from their tips into host tissue. How fungi undergo long-distance cell signalling to regulate effector production during infection is not known. Here we show that long-distance retrograde motility of early endosomes (EEs) is necessary to trigger transcription of effector-encoding genes during plant infection by the pathogenic fungus Ustilago maydis. We demonstrate that motor-dependent retrograde EE motility is necessary for regulation of effector production and secretion during host cell invasion. We further show that retrograde signalling involves the mitogen-activated kinase Crk1 that travels on EEs and participates in control of effector production. Fungal pathogens therefore undergo long-range signalling to orchestrate host invasion.

\footnotetext{
${ }^{1}$ School of Biosciences, College of Life and Environmental Sciences, University of Exeter, Stocker Road, Exeter EX4 4QD, UK. * These authors contributed equally to this work. $\dagger$ Present addresses: Department of Bioscience and Biotechnology, Faculty of Agriculture, Kyushu University, 6-10-1 Hakozaki, Fukuoka 812-8581, Japan (Y.H.); School of Geography, College of Life and Environmental Sciences, University of Exeter, Rennes Drive, Exeter EX4 4RJ, UK (N.S.). Correspondence and requests for materials should be addressed to G.S. (email: G.Steinberg@exeter.ac.uk).
} 
$\mathrm{P}$ lant pathogenic fungi invade their host plants by hyphal tip growth, which is an important determinant of fungal virulence. In response to infection, plant innate defences recognize pathogens and trigger a complex set of physiological responses 1,2 . Fungal pathogens overcome host immunity by secretion of a battery of effector proteins ${ }^{3-5}$. These effector proteins suppress plant immunity, thereby allowing rapid fungal colonization of plant tissue. Such interplay between pathogen and host forms the basis of the biotrophic interaction of the corn smut fungus Ustilago maydis and maize ${ }^{6}$. In this interaction, the fungus explores the plant surface by tip growth, but upon recognition of host surface cues initiates penetration of plant epidermal cells ${ }^{6,7}$. During this initial phase of development, a number of plant defence genes are induced, suggesting that the host recognizes the fungal intruder ${ }^{8}$. However, the host defence reaction is suppressed rapidly by secreted fungal effectors. A subset of these have been studied in depth, such as Pep1, required for penetration and for inhibition of apoplastic plant peroxidases ${ }^{9,10}$, Pit2, which inhibits apoplastic plant proteases ${ }^{11,12}$ and Cmu1, a chorismate mutase, which attenuates plant salicylic acid levels ${ }^{13}$ and thus suppresses plant defence responses.

As plant defence responses occur within minutes of perception of a pathogen ${ }^{14-16}$, fungal effector proteins must be produced and secreted equally rapidly. Effector production must therefore be induced as soon as the hyphal tip begins invasion. However, the architecture of an invading hyphal cell poses a challenge, because the nucleus is located at a considerable distance from the invading tip $^{17}$. It must therefore perceive a long-range signal from the plasma membrane to induce effector transcription. In animal neurons, such retrograde signalling from the synapse to the nucleus is mediated by early endosomes (EEs). They deliver signalling components, including mitogen-activated protein kinases (MAPKs; ref. 18) along microtubules to the nucleus ${ }^{19-21}$. Microtubule-dependent EE motility was described in $U$. maydis ${ }^{22,23}$ and other fungi ${ }^{24,25}$. Individual organelles can travel up to $90 \mu \mathrm{m}$, and therefore potentially traverse the distance between the hyphal tip and nucleus ${ }^{26,27}$. However, a role for fungal EEs in cell signalling has not been reported.

Here, we focus on the mechanism of long-range signalling during early infection of maize by $U$. maydis. We present evidence that effectors are secreted at the invading hyphal tip. We have developed a method to immobilize EEs very specifically, and we show that EE motility is crucial for transcription and subsequent secretion of the Cmu1, Pep1 and Pit2 effectors. EEs therefore transmit signals to the nucleus to regulate plant infection. Moreover, we provide evidence that the MAPK Crk1 localizes to moving EEs and that effector production and secretion is deregulated when crk1 is deleted. Collectively, our results are consistent with a role for motor-driven EE motility in retrograde signalling during infection. This long-range signalling mechanism is therefore of pivotal importance to fungal pathogenicity.

\section{Results}

Fungal EEs move retrogradely during plant infection. The corn smut fungus, $U$. maydis colonizes plant tissues using invasive hyphae (Fig. 1a) that grow at their tips. During infection, the a
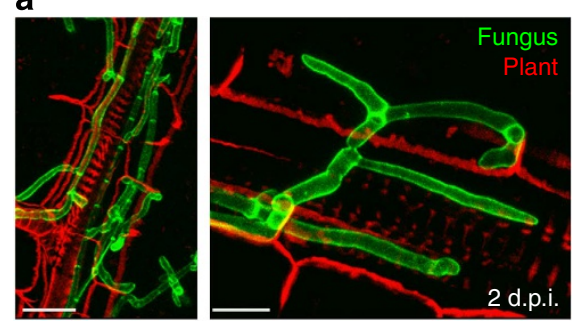

b

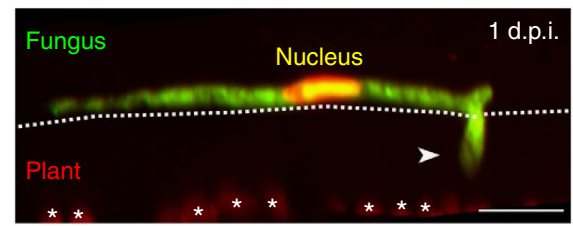

c

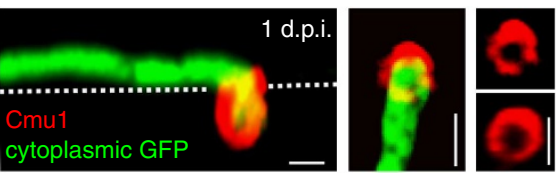

d
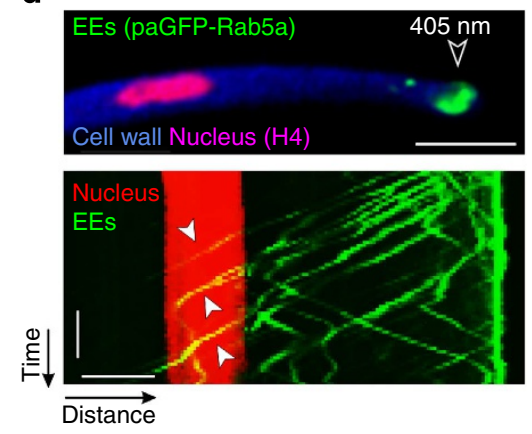

e

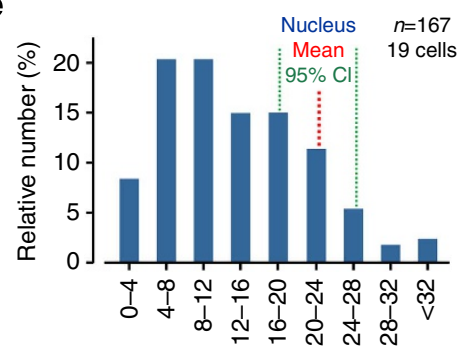

Retrograde run-length $(\mu \mathrm{m})$

Figure 1 | Endosomes travel from the invading hyphal tip to the nucleus. (a) Lectin-stained hyphal cells (green) colonizing a leaf at 2 d.p.i. Plant cell walls were stained using propidium iodide (red). Scale bars, $20 \mu \mathrm{m}$ (left panel) and $10 \mu \mathrm{m}$ (right panel). (b) Invasion of hyphal cells, expressing a nuclear RFP (nucleus; appearing yellow) and cytoplasmic GFP (green), at 1 d.p.i. Dotted line indicates plant surface. Chloroplasts visualized by their auto-fluorescence (asterisks, red). Arrow head points towards invading hyphal tip. Scale bar, $10 \mu \mathrm{m}$. See Supplementary Movie 1. (c) Secretion of effector protein Cmu1-mCherry (red) during early fungal invasion (fungus in green). Right panels reveal Cmu1-mCherry at the fungus-plant interface. Scale bars, $2 \mu \mathrm{m}$. See Supplementary Movie 2. (d) Image and kymograph show retrograde motility of photo-activated paGFP-Rab5a-labelled EEs (green) towards subapical nucleus (red, labelled with histone4-mCherry) in an invading fungal cell. The fungal cell wall was stained with Calcofluor White (blue). Point of photo-activation indicated by open arrowhead. Solid arrows in lower panel indicate long range motility. Scale bars, $10 \mu \mathrm{m}$ (upper panel), $3 \mathrm{~s}$ (vertical, lower panel) and $5 \mu \mathrm{m}$ (horizontal, lower panel). (e) Retrograde run-length of photo-activated EEs in invading hyphae (1 d.p.i.). Mean position of nucleus is indicated in red; $95 \%$ confidence intervals $(\mathrm{Cls})$ indicated by green dotted line. Sample size from $>3$ experiments is shown. 
Table 1 | Strains and plasmids used in this study.

\section{Strain or plasmid}

SG200

$\mathrm{SG} 200 \mathrm{G}_{3} \mathrm{NLSR}_{3}$

SG200Cmu1Ch_G

SG200Cmu1Ch_paGRab5a_H4Ch

AB33GRab5a

AB33GRab5a_cEAP

AB33Kin3G_ChRab5a_cEAP

SG200GRab5a

SG200GRab5a_cEAP

SG200GRab5a_mEAP

SG200GRab5a_cEAP_Cmu1Ch

SG200GRab5a_cEAP_Pep1Ch

SG200GRab5a_cEAP_APit2_nPit2Ch

SG200 $\Delta$ Kin3_GRab5a

AB33 $\Delta$ Kin3_ChRab5a_Kin3G

AB33G 3 Dyn2_ChRab5a

AB33G ${ }_{3}$ Dyn2_ChRab5a_cEAP

$\mathrm{AB} 33 \mathrm{Mcs} 1 \mathrm{G}_{3}$

AB33Mcs1G 3 _EAP

SG200crgG

SG200migG

SG200Cmu1Ch_G

SG200GRab5a_Pep1Ch_G

SG200G 3 nPit2Ch

SG200Cmu1Ch

AB33 $\Delta$ Hok 1

AB33GRab5a_AHok1

SG200 $\Delta$ Hok1

SG200nPit2Ch

SG200nPit2Ch_AHok1

SG200Cmu1Ch_ $\Delta$ Hok1

SG200Pep1Ch_ $\Delta$ Hok1

AB33 $\triangle$ Rab5a

AB33 $\triangle$ Rab5a_Yup1G

SG200 $\Delta$ Rab5a

SG200 $\Delta$ Rab5a_Cmu1Ch

SG200Pep1Ch

SG200 $\Delta$ Rab5a_Pep1Ch

SG200nPit2Ch__Rab5a

AB33mChRab5a_Kpp2G

AB33mChRab5a_Kpp4G

AB33mChRab5a_Fuz7G

AB33mChRab5a_Gsk3ßG

AB33mChRab5a_Kpp6G

AB33mChRab5a_Crk1G

AB33mChRab5a_Crk1G

$\mathrm{SG} 200 \mathrm{Crk1G}$

SG200cmu1G

SG200Cmu1Ch_ $\Delta$ Crk1

SG200cmu1G_ACrk1

SG200cmu1G_ $\Delta$ Hok1

$\mathrm{poG}_{3}$

poNLS3RFP

pNopaGRab5a

pCoH4Ch

$\mathrm{pHCmu1Ch}$

pCCmu1Ch

pHPep1Ch

pCPep1Ch

poGRab5a

pCoGRab5a

$\mathrm{po}_{\mathrm{m}} \mathrm{ChRab5a}$

$\mathrm{pHo}_{\mathrm{m}} \mathrm{ChRab5a}$

pKin3G

PHPcEAP

PCPCEAP

PCPMEAP

$\mathrm{p} \triangle \mathrm{Pit2}$

\section{Genotype}

a1 mfa2 bW2 bE1, ble

a1 $\mathrm{mfa} 2 \mathrm{bW} 2 \mathrm{bE1}, \mathrm{ble} \mathrm{e}^{R} / \mathrm{poG}_{3} / \mathrm{poNLS}_{\mathrm{RFP}}$

a1 mfa2 bW2 bE1, Pcmu1-cmu1-mcherry, ble ${ }^{R}$, hyg $^{R} / \mathrm{poG}_{3}$

a1 mfa2 bW2 bE1, Pcmu1-cmu1-mcherry, ble ${ }^{R}$, hyg ${ }^{R} / \mathrm{pNopaGRab5a/pCoH4Ch}$

a2 Pnar-bW2 Pnar-bE1, ble R $_{\text {poGRab5a }}$

a2 Pnar-bW2 Pnar-bE1, ble ${ }^{R} /$ poGRab5a/pHPcEAP

a2 Pnar-bW2 Pnar-bE1, Pkin3-kin3-egfp, ble ${ }^{R}, h g^{R} / \mathrm{po}_{\mathrm{m}} \mathrm{ChRab5a/pCPcEAP}$

a1 mfa2 bW2 bE1, ble ${ }^{R} /$ poGRab5a

a1 $\mathrm{mfa} 2$ bW2 bE1, ble $\mathrm{e}^{R} / \mathrm{poGRab} 5 \mathrm{a} / \mathrm{pHPcEAP}$

a1 mfa2 bW2 bE1, ble ${ }^{R} /$ poGRab5a/pCPmEAP

a1 mfa2 bW2 bE1, Pcmu1-cmu1-mcherry, ble $e^{R}, c b x^{R} /$ poGRab5a/pHPcEAP

a1 mfa2 bW2 bE1, Ppep1-pep1-mcherry, ble ${ }^{R}, c b x^{R} /$ poGRab5a/pHPcEAP

a1 mfa2 bW2 bE1, $\Delta$ pit2, ble ${ }^{R}, \mathrm{G} 418^{R} / \mathrm{poGRab5a/pCnPit2Ch}$

a1 mfa2 bW2 bE1, $\Delta$ kin3, ble ${ }^{R}$, nat $^{R} /$ pCoGRab5a

a2 Pnar-bW2 Pnar-bE1, $\Delta k i n 3, b l e^{R}$, nat $^{R} / \mathrm{pHo}_{\mathrm{m}} \mathrm{ChRab5a/pKin3G}$

a2 Pnar-bW2 Pnar-bE1, Pdyn2-3xegfp-dyn2, ble ${ }^{R}$, hyg ${ }^{R} / \mathrm{po}_{\mathrm{m}} \mathrm{ChRab5a}$

a2 Pnar-bW2 Pnar-bE1, Pdyn2-3xegfp-dyn2, ble ${ }^{R}, h_{y g}{ }^{R} / \mathrm{po}_{m}$ ChRab5a/pCPcEAP

a2 Pnar-bW2 Pnar-bE1, Pmcs1-mcs1-3xegfp, ble ${ }^{R}, h_{y g}{ }^{R}$

a2 Pnar-bW2 Pnar-bE1, Pmcs1-mcs1-3xegfp, $b e^{R}$, hyg $^{R} /$ pCPcEAP

a1 $\mathrm{mfa} 2$ bW2 bE1, ble $\mathrm{R}^{\prime} / \mathrm{pcrg} 1 \mathrm{GFP}$

a1 mfa2 bW2 bE1, ble ${ }^{R} /$ pmig1GFP

a1 mfa2 bW2 bE1, Pcmu1-cmu1-mcherry, ble ${ }^{R}$, hyg $^{R} / \mathrm{poG}_{3}$

a1 mfa2 bW2 bE1, Ppep1-pep1-mcherry, ble ${ }^{R}$, hyg $R /$ poGRab5a/poG 3

a1 $\mathrm{mfa} 2 \mathrm{bW} 2 \mathrm{bE1}, \mathrm{ble} / \mathrm{poG}_{3} / \mathrm{pHnPit} 2 \mathrm{Ch}$

a1 mfa2 bW2 bE1, Pcmu1-cmu1-mcherry, ble ${ }^{R}$, hyg ${ }^{R}$

a2 Pnar-bW2 Pnar-bE1, $\Delta$ hok1, ble ${ }^{\mathrm{R},}$, hyg ${ }^{\mathrm{R}}$

a2 Pnar-bW2 Pnar-bE1, $\Delta$ hok1, ble ${ }^{\prime}, h^{\prime} g^{R} /$ poGRab5a

a1 mfa2 bW2 bE1, $\Delta$ hok1, ble ${ }^{R}$, hyg $^{R}$

a1 $\mathrm{mfa} 2 \mathrm{bW} 2 \mathrm{bE1}, \mathrm{ble} \mathrm{R}^{R} / \mathrm{pHnPit} 2 \mathrm{Ch}$

a1 mfa2 bW2 bE1, $\Delta$ hok1, ble ${ }^{R}$, nat $^{R} / \mathrm{pHnPit} 2 \mathrm{Ch}$

a1 mfa2 bW2 bE1, Pcmu1-cmu1-mcherry, $\Delta$ hok1, ble ${ }^{R}$, hyg $^{R}$, nat $^{R}$

a1 mfa2 bW2 bE1, Ppep1-pep1-mcherry, $\Delta$ hok1, ble ${ }^{R}$, hyg $^{R}$, nat $^{R}$

a2 Pnar-bW2 Pnar-bE1, $\Delta r a b 5 a, b l e^{R}$, nat $^{R}$

a2 Pnar-bW2 Pnar-bE1, $\Delta r a b 5 a, b l e^{R}, n^{R} t^{R} /$ PYup1SG2

a1 mfa2 bW2 bE1, $\Delta r a b 5 a, b l e^{R}, n a t^{R}$

a1 mfa2 bW2 bE1, $\Delta$ rab5a, Pcmu1-cmu1-mcherry, ble ${ }^{R}$, nat $^{R}$, hyg $^{R}$

a1 mfa2 bW2 bE1, Ppep1-pep1-mcherry, ble ${ }^{R}$, hyg $^{R}$

a1 mfa2 bW2 bE1, $\Delta$ rab5a, Ppep1-pep1-mcherry, ble ${ }^{R}$, nat $^{R}$, hyg $^{R}$

a1 mfa2 bW2 bE1, $\Delta$ rab5a, ble ${ }^{R}$, nat $^{R} / \mathrm{pHnPit} 2 \mathrm{Ch}$

a2 Pnar-bW2 Pnar-bE1, ble $/$ po $_{m}$ ChRab5a/pKpp2G

a2 Pnar-bW2 Pnar-bE1, ble $/$ po $_{m}$ ChRab5a/pKpp4G

a2 Pnar-bW2 Pnar-bE1, ble $/$ po $_{m}$ ChRab5a/pFuz7G

a2 Pnar-bW2 Pnar-bE1, ble ${ }^{R} /$ po $_{m}$ ChRab5a/pGsk3ßG

a2 Pnar-bW2 Pnar-bE1, ble $/$ po $_{m}$ ChRab5a/pKpp6G

a2 Pnar-bW2 Pnar-bE1, ble $\mathrm{R} / \mathrm{po}_{\mathrm{m}} \mathrm{ChRab5a/pCrk1G}$

a2 Pnar-bW2 Pnar-bE1, Pcrk1-crk1-3xegfp, ble ${ }^{R}$, hyg ${ }^{R} / \mathrm{po}_{\mathrm{m}} \mathrm{ChRab5a}$

a1 mfa2 bW2 bE1, Pcrk1-crk1-3xegfp, ble ${ }^{\mathrm{R}}$, hyg ${ }^{R}$

a1 mfa2 bW2 bE1, ble $/$ pcmu1GFP

a1 mfa2 bW2 bE1, Pcmu1-cmu1-mcherry, $\Delta c r k 1, b l e^{R}, h y g^{R}, G 418^{R}$

a1 mfa2 bW2 bE1, $\Delta c r k 1, b l e^{R}, G 418^{R} / \mathrm{pcmu1GFP}$

a1 mfa2 bW2 bE1, $\Delta$ hok1, ble ${ }^{R}, h y g^{R} /$ pcmu1GFP

Potef-3xegfp, $c b x^{R}$

Potef-gal4s-3xmrfp, nat ${ }^{R}$

Potef-pagfp-rab5a, nat ${ }^{R}$

Potef-his4-mcherry, $c b x^{R}$

Pcmu1-cmu1-mcherry, hyg ${ }^{R}$

Pcmu1-cmu1-mcherry, $c b x^{R}$

Ppep1-pep1-mcherry, hyg ${ }^{R}$

Ppep1-pep1-mcherry, $c b x^{R}$

Potef-egfp-rab5a, nat ${ }^{R}$

Potef-egfp-rab5a, cbx ${ }^{R}$

Potef-mcherry-rab5a, nat ${ }^{R}$

Potef-mcherry-rab5a, hyg ${ }^{R}$

Pkin3-kin3-egfp, $c b x^{R}$

Pcrg1-kin1 ${ }^{\text {G96E, }}$-739-PX, hyg ${ }^{R}$

Pcrg1-kin1 G96E,1-739-PX, cbx ${ }^{R}$

Pmig1-kin1G96E,1-739-PX,cbx ${ }^{R}$

$\Delta$ pit2, G418 ${ }^{R}$
Reference

Ref. 35

This study

This study

This study Ref. 64

This study

This study

This study

This study

This study

This study

This study

This study

This study

Ref. 27

Ref. 27

This study

This study

This study

This study

This study

This study

This study

This study

This study

Ref. 37

Ref. 37

This study

This study

This study

This study

This study

This study

This study

This study

This study

This study

This study

This study

This study

This study

This study

This study

This study

This study

This study

This study

This study

This study

This study

This study

This study

Ref. 27

This study

This study

This study

This study

This study

This study

Ref. 64

Ref. 65

Ref. 27

Ref. 33

Ref. 69

This study

This study

This study

This study 


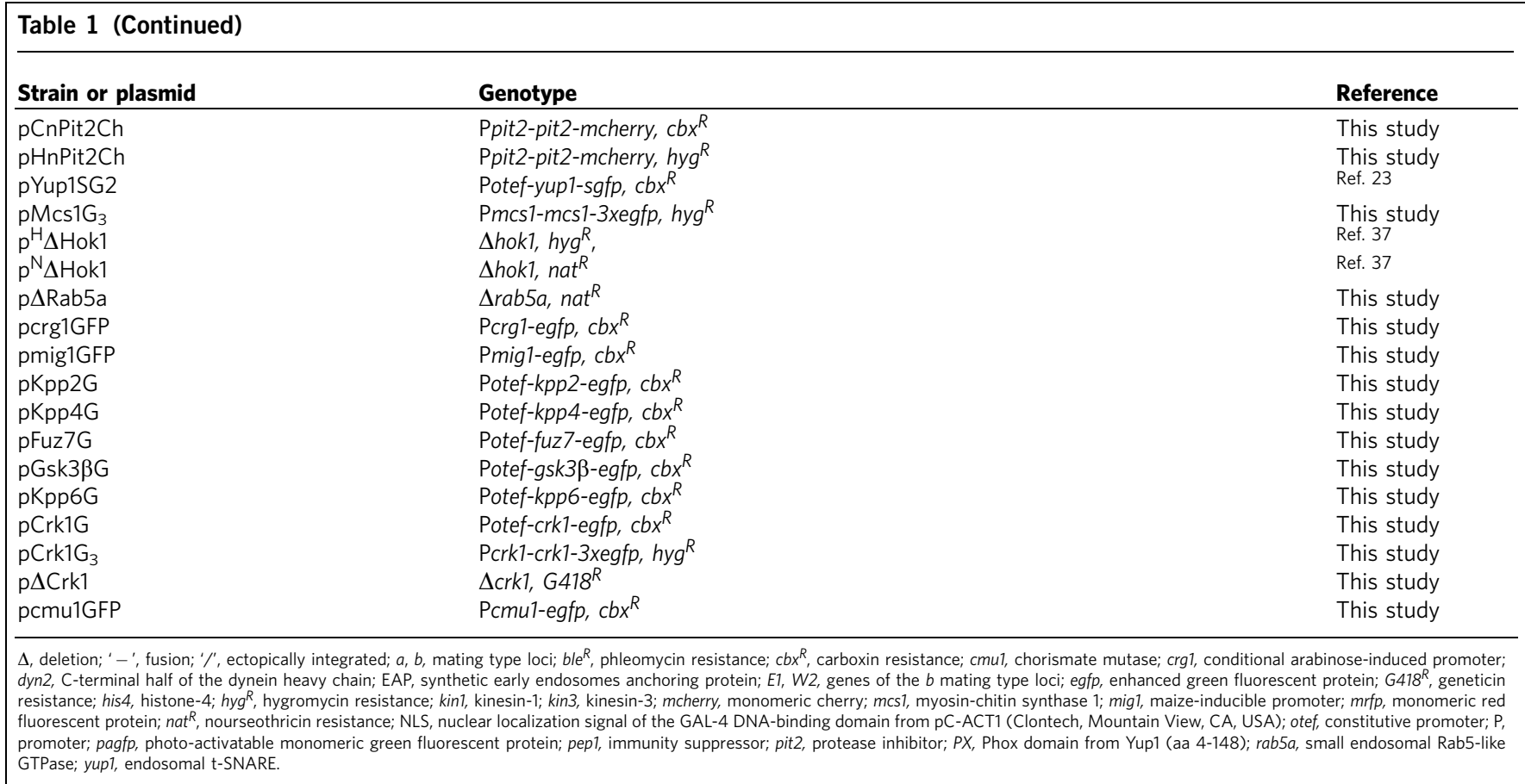

fungus secretes a battery of effector proteins ${ }^{9,11-13,28,29}$. These effector proteins allow the pathogen to remain masked from plant recognition, thereby overcoming plant defence. We visualized the fungal nucleus, during the initial phase of plant infection, by expressing triple red fluorescent protein (RFP) fused to a nuclear localization signal in cells that also expressed cytoplasmic green fluorescent protein (GFP; for all genotypes see Table 1; and for usage of strains see Supplementary Table 1). We found the nucleus routinely located $22.00 \pm 2.01 \mu \mathrm{m}$ behind the distal invading tip (Fig. $1 \mathrm{~b}$ and Supplementary Movie 1). To investigate effector secretion, we visualized the metabolic effector chorismate mutase Cmul (ref. 13). We tested whether Cmul is secreted upon host penetration by fusing mCherry to the endogenous cmul gene and expressing the translational fusion in a $U$. maydis strain that co-expressed cytoplasmic GFP. This did not impair virulence, demonstrating that the fusion protein is fully functional (Supplementary Fig. 1a,b). Cmul surrounded the invading hyphal tip (Fig. 1c), consistent with its role in suppressing plant defence ${ }^{13}$. Significantly, this occurred at a long distance from the nucleus of the invading hypha that remained on the leaf surface (Fig. $1 \mathrm{~b}$ and Supplementary Movie 1). We then tested whether EE motility might provide the mechanism for long-range signalling between the invading hyphal tip and nucleus. For this, we co-expressed a photoactivatable EE marker paGFP-Rab5a (ref. 26) and mCherryhistone-4 in invasive hyphae in $U$. maydis. Following laser-based photo-activation, EEs became visible and travelled rapidly from the hyphal tip towards the nucleus (Supplementary Movie 2 and Fig. 1d, lower panel shows kymograph, in which motility is represented by diagonal lines). We found that $36 \%$ of signals $(n=167)$ reached or passed the nucleus (Fig. 1e), demonstrating that retrograde EE motility could mediate communication between the hyphal tip and nucleus during infection.

A synthetic protein blocks specifically EE motility. Bi-directional EE motility in U. maydis is driven by the motor protein kinesin-3 (Kin3, ref. 26). We found that a $\Delta k i n 3$ mutant was less virulent (Supplementary Fig. 2a,b), suggesting that EE motility is important for infection. However, Kin3 has been implicated in secretion in $U$. maydis ${ }^{30}$ and it is possible, therefore, that inhibition of an EE-independent kinesin-3 function attenuates virulence. To investigate this possibility, we designed a synthetic protein to immobilize EEs more specifically, without blocking kinesin-3 motility. We used an EE anchoring protein (EAP) consisting of a non-motile kinesin-1 motor head ${ }^{31}$, fused to the EE-binding Phox domain of t-SNARE Yup1 (ref. 23; Fig. 2a). The EAP construct was expressed under the inducible crg1 promoter ${ }^{32}$, which is glucose-repressible and strongly-inducible by arabinose, and transformed into strains expressing GFP-Rab5a-labelled EEs. In the absence of the synthetic protein, normal EE motility was observed (Fig. 2b,d; no EAP), but expression of the chimaeric EAP strongly inhibited EE movement (Fig. 2b,d; + EAP; Supplementary Movie 3). Expression of EAP did not inhibit EEindependent motility of fluorescent Kin3 in yeast or hyphal cells (Fig. 2c,e; Supplementary Movie 4) or fluorescent dynein (Fig. 2f-h), nor did it inhibit apical localization of chitin synthase Mcs1 (Fig. 2i,j), which is dependent on the Kin1 motor ${ }^{33}$. EAP did not therefore affect dynein, kinesin-1 or kinesin-3-mediated transport along microtubules. As these motors mediate the majority of the intracellular transport in $U$. maydis ${ }^{30}$, we conclude that EAP specifically blocks EE motility, but without inhibition of other motor-driven intracellular trafficking.

EE motility is essential for early plant infection. Having established EAP as a specific tool to block EE motility, we investigated EE motility during early and late plant colonization. EAP was expressed under control of the $\operatorname{crg} 1$ - and plant-induced mig1 promoter, which is repressed during early plant invasion ${ }^{34}$. We confirmed the expression pattern of both promoters by monitoring cytoplasmic GFP in planta. The crg1 promoter induces GFP expression during early stages of infection and is repressed after 2-3 days, whereas mig1-driven expression is observed at 3 days post infection (d.p.i.) onwards (Supplementary Fig. 3).

To test the role of EE motility in plant infection, we generated SG200 strains, expressing GFP-Rab5a and two mutants that 
a
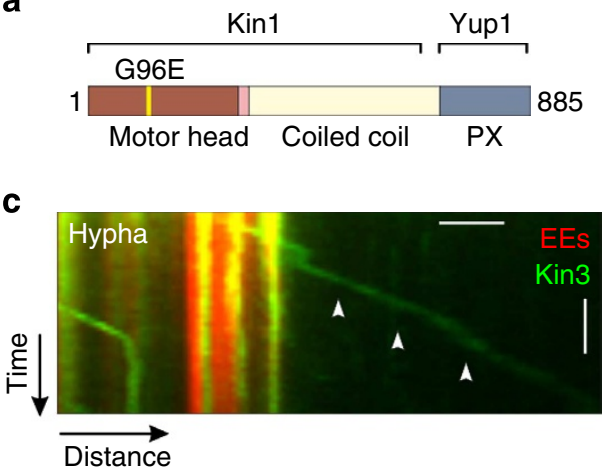

d

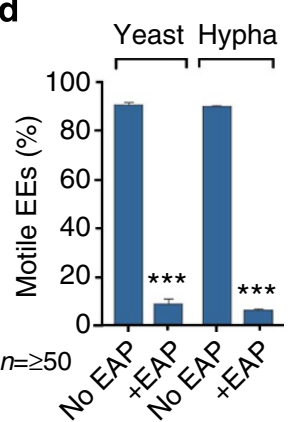

h

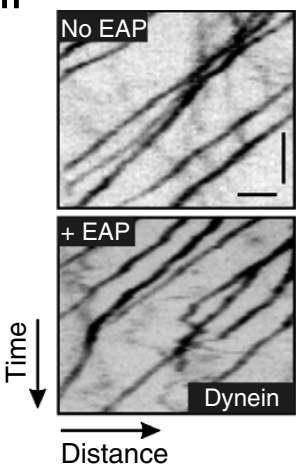

e

$$
\text { }
$$

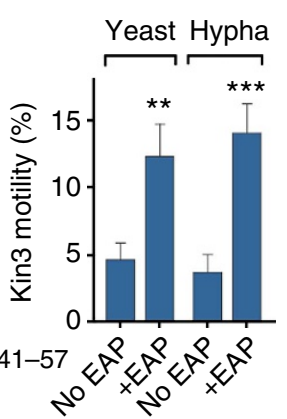

i

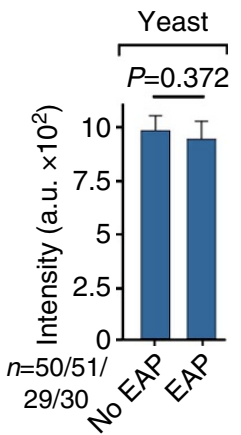

b

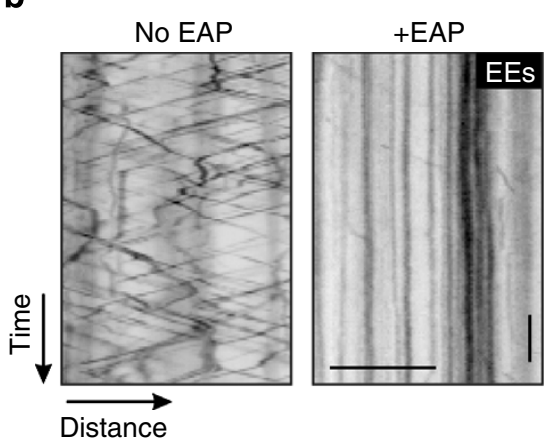

f

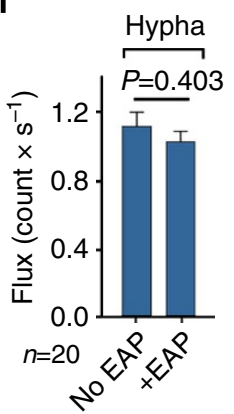

g

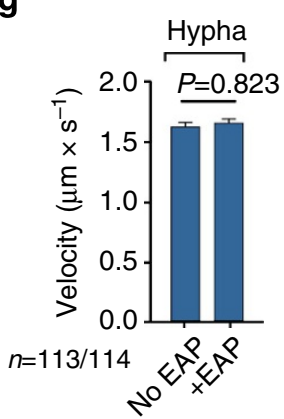

j

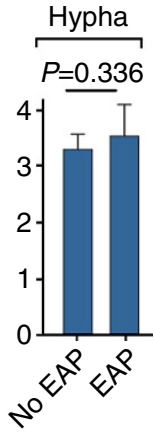

+ EAP

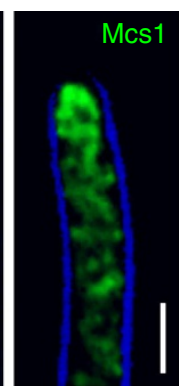

Figure 2 | A synthetic anchorage protein blocks fungal EE motility. (a) Organization of synthetic linker EAP; N-terminal part of an immobile kinesin-1 ${ }^{\text {rigour }}$ protein (aa 1-739) fused to an EE-binding Phox domain (PX) of the putative t-SNARE Yup1 (ref. 23). (b) Contrast-inverted kymographs showing motility of GFP-Rab5a-labelled EEs. Cells were pre-grown in glucose-containing (no EAP) or arabinose-containing medium (+ EAP). The linker blocks EE motility. Scale bars, $3 \mathrm{~s}$ (vertical) and $3 \mu \mathrm{m}$ (horizontal). See Supplementary Movie 3. (c) Kymograph showing motility of kinesin-3-GFP (green, arrowheads), unbound to stationary EEs (red) in a hyphal cell expressing EAP for $5 \mathrm{~h}$. Scale bars, $2 \mathrm{~s}$ (vertical) and $2 \mu \mathrm{m}$ (horizontal). See Supplementary Movie 4. (d) Motile EEs in yeast-like (yeast) and hyphal cells (hypha) in absence (no EAP) or presence of EAP expression ( + EAP). Mean \pm s.e. and sample size from two experiments is shown. ${ }^{\star \star \star}$ Significance at $P<0.001$, unpaired Student's $t$-test. (e) EE-independent movement of GFP-labelled kinesin-3 motors in the presence ( + EAP) or absence of EAP (no EAP) in yeast-like cells and hyphae. Mean \pm s.e. and sample size $n$ from two to three experiments is shown. ${ }^{\star *}$ Significance at $P=0.0066,{ }^{\star \star *}$ significance at $P=0.0004$, unpaired Student's $t$-test. (f) Flux of dynein in the presence ( + EAP) or absence of EAP (no EAP). Mean \pm s.e. and sample size $n$ from two experiments is shown. No significant difference at $P=0.4030$, unpaired Student's $t$-test. (g) Velocity of retrograde dynein in the presence ( + EAP) or absence of EAP (no EAP). Mean \pm s.e. and sample size $n$ from two experiments is shown. No significant difference at $P=0.823$, unpaired Student's $t$-test. (h) Contrast-inverted kymographs showing retrograde motility of dynein in the absence of EAP (no EAP) and after $15 \mathrm{~h}$ of expression of EAP ( + EAP). Scale bars, $1 \mathrm{~s}$ (vertical) and $1 \mu \mathrm{m}$ (horizontal). (i) Apical Mcs1-GFP ${ }_{3}$ signal intensity at the growth region of yeast-like and hyphal cells in the presence $(+E A P)$ or absence of EAP (no EAP). Mean \pm s.e. and sample size $n$ from one experiment is shown. The result was confirmed by a non-quantitative experiment. No significant difference at an error probability of $P=0.372$ and 0.336 , unpaired Student's t-test. (j) Apical Mcs1-GFP 3 at the growth region of hyphal cells in the presence (+EAP) or absence of EAP (no EAP). Scale bar, $2 \mu \mathrm{m}$.

contained EAP under the crg1 promoter (crg1EAP) or mig1 promoter (mig1EAP). SG200 strains are widely used in virulence studies in $U$. maydis ${ }^{9,13,29}$, because they are able to auto-induces a pheromone cascade, which initiates hyphal growth on charcoal-containing agar medium and during plant infection without the need for fusion of two compatible cells ${ }^{35}$. Using these strains we observed EEs during plant tissue invasion (Fig. 3a and Supplementary Movie 5). In control experiments, EEs moved bidirectionally throughout infection (Fig. 3b,c and Supplementary Movie 5; control). When plants were infected with crglEAP, pre-grown in arabinose-medium, EE motility was blocked for the first 2 days but re-established when 
a

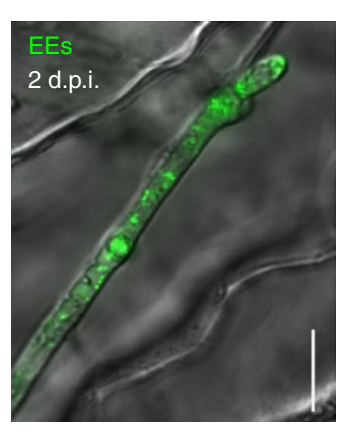

b

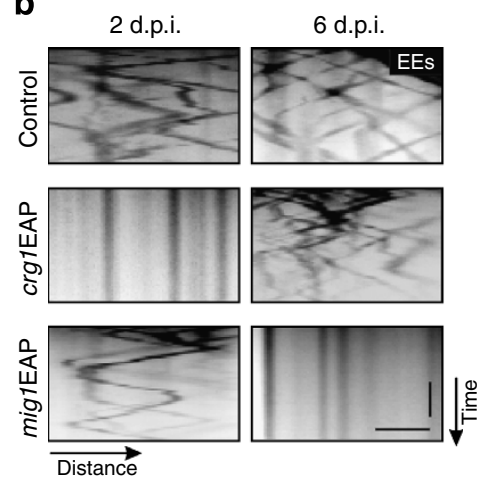

C

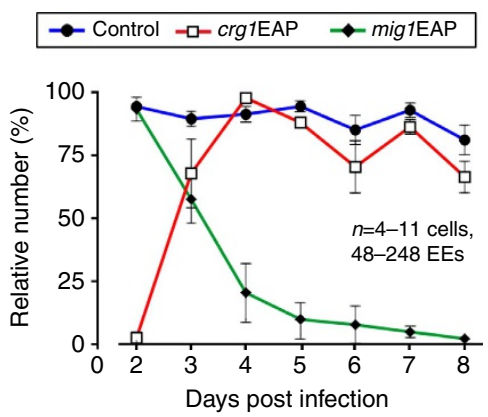

f

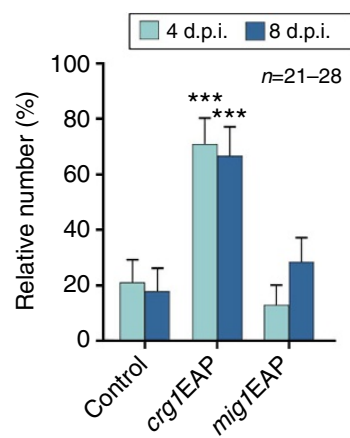

g

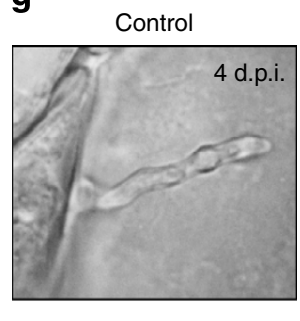

$\operatorname{crg} 1 \mathrm{EAP}$

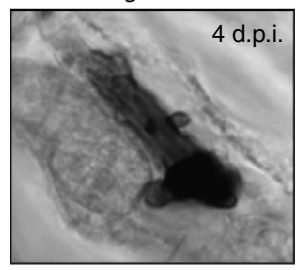

mig1EAP

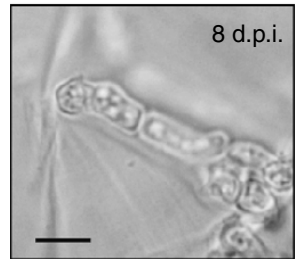

d

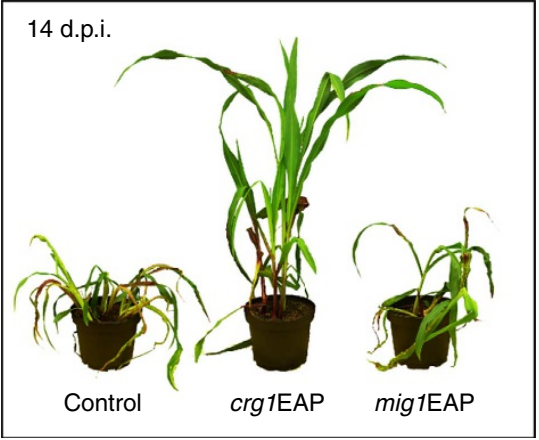

e

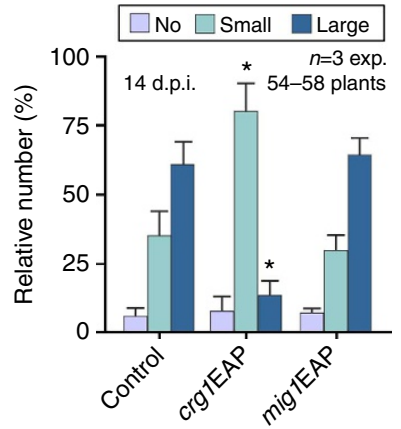

Figure 3 I EE motility is essential for early plant infection. (a) GFP-Rab5a-labelled EEs in invasive fungal cell (2 d.p.i.). Scale bar, $10 \mu$ m. See Supplementary Movie 5. (b) Contrast-inverted kymographs showing EE motility in invasive hyphae. Expression of EAP under crg1 promoter blocks EE motility at 2 d.p.i., whereas mig1 promoter-driven expression inhibits motility at 6 d.p.i. Scale bars, $3 \mathrm{~s}$ (vertical) and $1 \mu$ m (horizontal). See Supplementary Movie 6. (c) Graph showing EE motility during pathogenic development. Mean \pm s.e. and sample size $n$ from one experiment is shown. The result was confirmed by a non-quantitative experiment. (d) Maize infected with wild-type U. maydis (control) and mutants that block EE motility during early infection (crg1EAP) and late infection (mig1EAP) at 14 d.p.i. (e) Disease symptoms post infection with control, crg1EAP and mig1EAP. Mean \pm s.e.; sample sizes

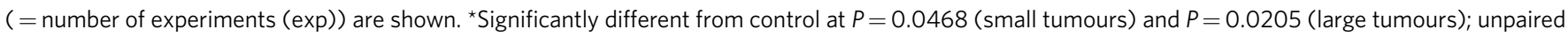
Student's t-test. No tumours: anthocyanin and chlorosis but no swellings; small tumours: swellings $<3$ mm; large tumours: swellings $>3$ mm.

(f) Diaminobenzidine precipitation indicates plant defence-associated $\mathrm{H}_{2} \mathrm{O}_{2}$ in leaves infected with wild type, crg1EAP and mig1EAP. Mean \pm s.e. and sample sizes from one experiment is shown. The result was confirmed by a non-quantitative experiment. ${ }^{\star \star \star}$ Significantly different from control at $P=0.0003$ ( 4 d.p.i.) and $P=0.0009$ ( 8 d.p.i.); unpaired Student's $t$-test. (g) Diaminobenzidine precipitation in control, crg1EAP and mig1EAP mutants. Scale bar, $5 \mu$ m.

the crg1 promoter was glucose-repressed at later time points (Fig. 3b,c and Supplementary Movie 6). By contrast, EE motility was initially normal in the mig1EAP strain, but blocked 3-4 days after infection (Fig. 3b,c and Supplementary Movie 6). Thus, by placing EAP under control of different promoters, we established an infection-stage-specific means of inhibiting EE motility.

We then investigated the role of EE motility during early and late plant infection. Inoculation of maize plants with control strain or mig1EAP caused disease symptoms, including inhibition of plant growth (Fig. 3d) and 'tumour' formation (Fig. 3e). We therefore conclude that EE motility is not essential at late stages of infection. However, infection with crg1EAP led to severe attenuation of disease symptoms (Fig. 3d,e). Blocking EE motility during early infection therefore strongly reduced virulence of the fungus. We reasoned that this might be owing to an inability to suppress plant immunity. One early defence reaction of the plant is to release $\mathrm{H}_{2} \mathrm{O}_{2}$ during an oxidative burst, which can be visualized by precipitation of diaminobenzidine ${ }^{36}$. We stained infected leaves with diaminobenzidine and found little evidence of an oxidative burst in wild-type or mig1EAP infections at 4-8 d.p.i. (Fig. 3f,g), which is consistent with suppression of early immune responses during successful $U$. maydis infection ${ }^{9,12,13}$. By contrast, crg1EAP strains induced an oxidative burst
(Fig. 3f,g). We conclude that EE motility is required to suppress host defences during early stages of infection.

Endosome motility is required for fungal effector production. Suppression of plant immunity responses suggested the EE motility may be necessary for fungal effector production and secretion. To test this idea, we expressed functional gene fusions of mCherry and Cmul (ref. 13), the immunity suppressor Pep1 (ref. 9) and the protease inhibitor Pit2 (ref. 12), and observed their secretion in planta. Fungi expressing Cmu1-mCherry, Pep1mCherry caused strong disease symptoms (Supplementary Fig. 1a,b), indicating that the fusion protein is fully functional. In contrast, Pit2-mCherry producing strains showed attenuated virulence (Supplementary Fig. 1a,b). Each effector accumulated in the apoplastic space (Supplementary Fig. 1c-e), as previously reported $^{9,12,13}$. We then infected plants with $\operatorname{crg} 1$ EAP strains coexpressing each fluorescent effector protein and the EE marker GFP-Rab5a. When cells were pre-grown in glucose-containing media, infecting fungi showed EE motility and normal secretion of fluorescent effectors (Fig. 4a, left panels; lower left kymographs show EE motility by diagonal lines). When cells were grown in inducing (arabinose-containing) media, EAP was produced and EE motility was inhibited (Fig. 4a, right panels; lower right 
a

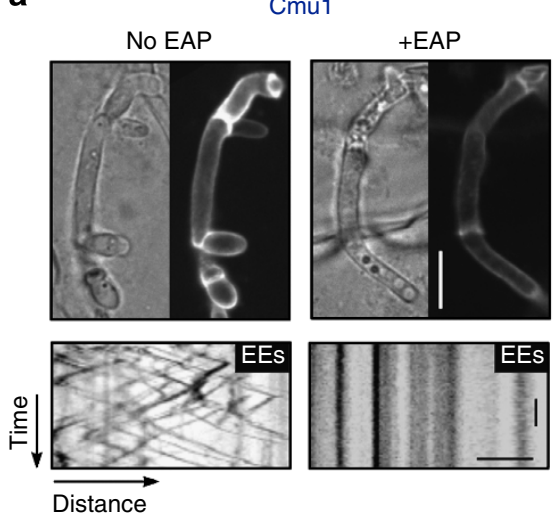

PeP1

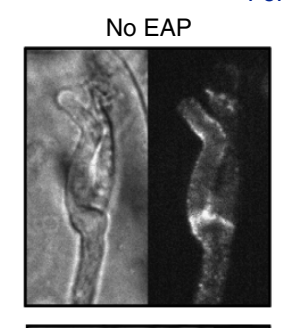

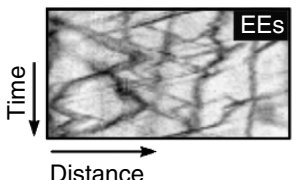
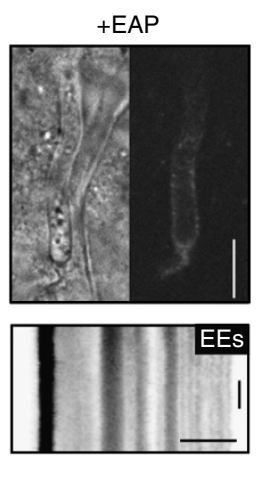

Pit2

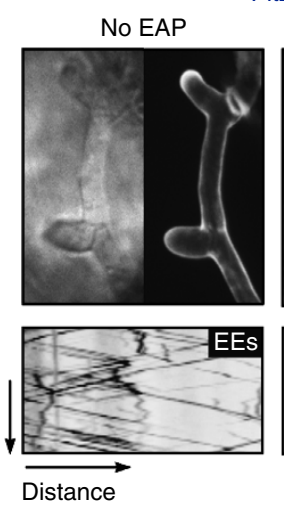

+ EAP

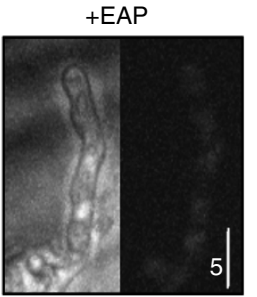

b

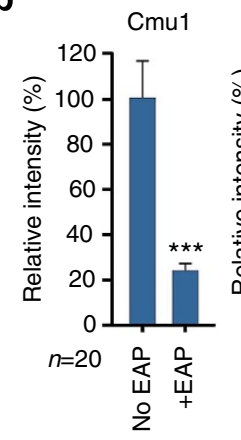

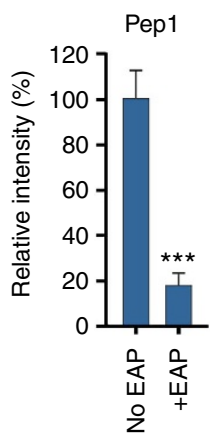

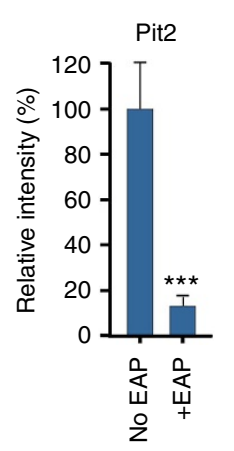

C

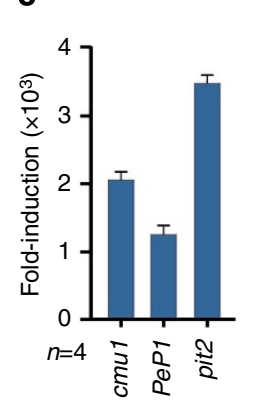

d

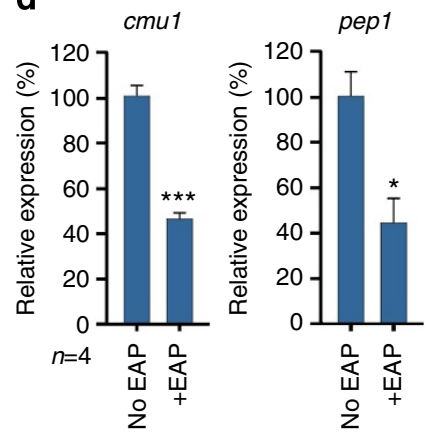

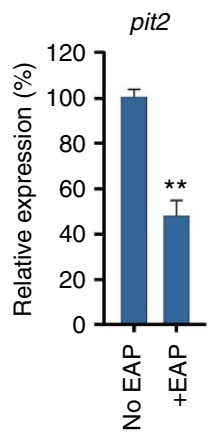

Figure 4 | EE motility is required for effector secretion and transcription of effector-encoding genes in $\boldsymbol{U}$. maydis (a) Impaired secretion of effectors during infection. Cmu1-mCherry (Cmu1), Pep1-mCherry (Pep1) or Pit2-mCherry (Pit2) expressed in cells that contain EAP, which impaired EE motility (contrast-inverted kymographs, stationary signals appear as vertical lines). Cells were pre-grown in glucose- (no EAP) and arabinose-containing medium (+EAP). Scale bars, $5 \mu \mathrm{m}$ (upper panels), $3 \mathrm{~s}$ (vertical, lower panels) and $2 \mu \mathrm{m}$ (horizontal, lower panels). (b) Secretion of fluorescent protein-effector fusions in plants at 2 d.p.i. Mutants were pre-grown in glucose- (no EAP) and arabinose-containing medium ( + EAP). Mean \pm s.e. and sample size from one experiment is shown. The result was confirmed by a non-quantitative experiment. ${ }^{\star \star \star}$ Significance at error probability $P_{\mathrm{cmu} 1}$ and $P_{\text {pit2 } 2}=0.0002$, $P_{\text {pep } 1}<0.0001$; unpaired Student's t-test. Signals were corrected by subtracting intracellular fluorescent background. (c) Induction of fungal effector transcription during in plant infection. Expression in planta at 1 d.p.i. was compared with transcript levels in liquid culture. Mean \pm s.e. and sample sizes ( = number of experiments) are shown. (d) Relative transcript levels of cmu1, pep1 and pit2 genes in infectious fungal cells grown at 1-2 d.p.i. Cells were pre-grown in glucose- (no EAP) and arabinose-containing medium (+EAP). See Supplementary Methods section. Expression of effector genes in control cells was set to $100 \%$. Mean \pm s.e. and sample sizes ( $=$ number of experiments) are shown. ${ }^{\star}$ Different from control at $P_{\text {pep } 1}=0.0149$, ${ }^{\star \star}$ different from control at $P_{\text {pit2 }}=0.0026$ and ${ }^{\star \star \star}$ different from control at $P_{\text {cmul }}=0.0009$; unpaired Student's $t$-test.

kymograph shows the absence of motility). Importantly, in these cells, the peripheral signal of all three effector proteins was decreased markedly (Fig. 4a,b and Supplementary Fig. 1c-e), suggesting that a block in EE motility impairs effector secretion. This defect in effector secretion raised the possibility that retrograde EE-based signalling is required to induce effector gene transcription at the nucleus. We therefore measured transcript levels of cmu1, pep1 and pit2 using quantitative realtime PCR (qRT-PCR). None of the effectors was expressed in axenic culture, whereas effector expression was strongly induced in planta when EAP was not present (Fig. 4c; bars show induction compared with liquid culture). However, when EE motility was blocked by expression of EAP under the $\operatorname{crg} 1$ promoter, we observed significantly reduced effector transcription (Fig. 4d, + EAP; error probability $P_{\mathrm{cmu} 1}: 0.0009 ; P_{\mathrm{pep} 1}: 0.0149$ and $P_{\text {pit } 2}$ : 0.0026; unpaired Student's $t$-test). We conclude that effector transcription and, consequently, effector secretion depends on EE motility.

Many signalling pathways are located to EEs, including phosphatidylinositol 3-phosphate (PtdIns3P) signalling ${ }^{19}$. The Phox domain of EAP is expected to bind to PtdIns3P, and thus might have unrecognized side effects. We therefore set out to find EAP-independent ways of blocking EE motility and the putative retrograde signalling mechanism. Recently, the orthologous hook proteins Hok 1 and HookA, identified, respectively, in U. maydis ${ }^{37}$ and Aspergillus nidulans ${ }^{38}$, were shown to act as adaptors that link microtubule-dependent motors to EEs. They are thus crucial for fungal EE motility, and we set out to investigate effector transcription and secretion in $\Delta h o k 1$ mutants. We found that hok1 null mutants were still able to generate hyphal cells (Supplementary Fig. 4), demonstrating that pheromone signalling pathways were not affected in this strain. Consistent with previous reports, these hyphae remained short, and we observed immobile EEs clustered at their cell ends (Fig. 5a,b) (ref. 37). Similar to EAP-expressing strains, hok1 null mutants were impaired in their ability to cause disease (Fig. 5c,d). Most strikingly, the transcription and secretion of all tested effectors was reduced significantly in hok1 null mutants (Fig. 5e-g). These results are therefore consistent with our conclusion that EE motility is required for the induction of effector production and exocytosis. To test this hypothesis further, we deleted the fungal homologue of the small GTPase Rab5 (ref. 39), which is of central importance for EE function in mammalian cells ${ }^{40}$. In the absence of Rab5a, short hyphae formed (Fig. 6a), containing largely immobile EE accumulations at the ends of cells (Fig. 6b), which were labelled by the EE marker Yup1-GFP (ref. 23). Similar to 
a

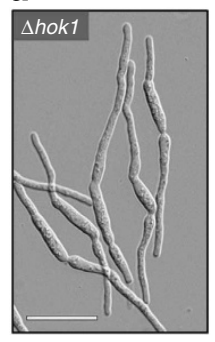

e

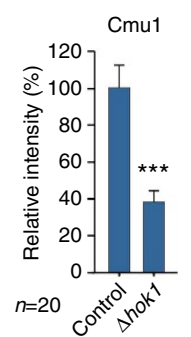

b

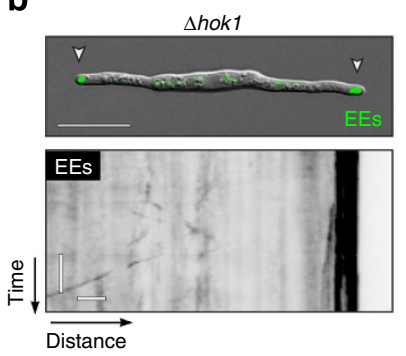

c

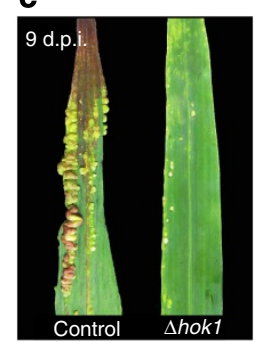

d

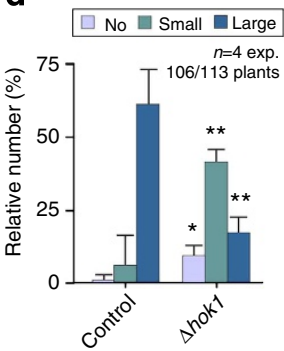

f

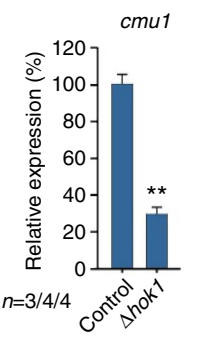

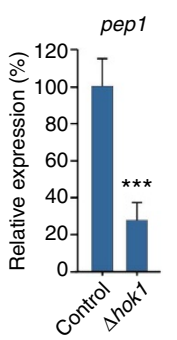

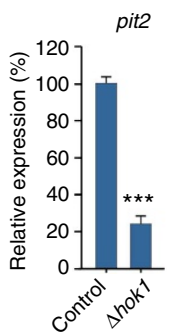

g
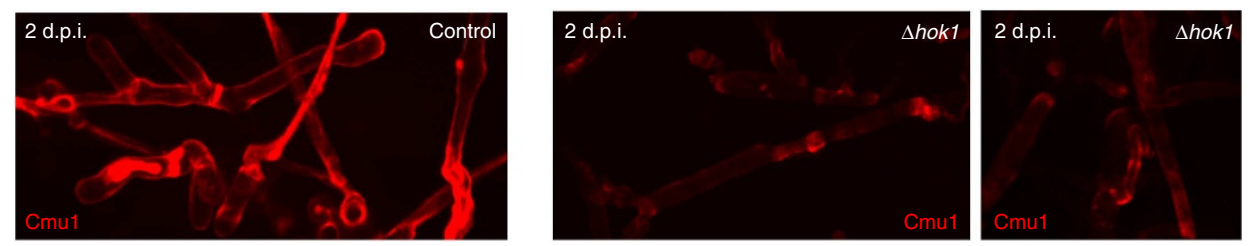

Figure 5 | Effector secretion and pathogenicity are impaired in hok1 null mutants. (a) Phenotype of hok1 deletion mutants. Scale bar, $20 \mu \mathrm{m}$. (b) EE localization and motility in hok1 deletion mutants. Upper panel shows EE clusters (GFP-Rab5a, green, arrowheads) at the poles of the short mutant cells. Lower kymograph shows the absence of EE motility in $\Delta$ hok1 mutants. Scale bars, $20 \mu \mathrm{m}$ (upper panel), $3 \mathrm{~s}$ (vertical, lower panel) and $1 \mu \mathrm{m}$ (horizontal, lower panel). (c) Plant disease symptoms at 9 days after infection of maize plants with control and $\Delta$ hok 1 strains. (d) Plant symptoms at 14 days after infection with control and hok1 deletion mutants. Mean \pm s.e. and sample sizes $n$ (=number of experiments (exp)) are shown. ${ }^{\star} D i f f e r e n t$ from control at error probability $P=0.019$; ${ }^{\star \star}$ different from control at error probability $P=0.0047$; unpaired Student's $t$-test. (e) Secretion of fluorescent effectors,

indicated by the signal intensity at the periphery of the invasive hyphae in plants at 2 d.p.i. Mean \pm s.e. and sample sizes from one experiment are shown. The result was confirmed by a non-quantitative experiment. ${ }^{\star}$ Different from control at $P_{\text {pep } 1}=0.0184$, ${ }^{\star \star \star}$ different from control at $P_{\text {cmu1 }}=0.0002$ and $P_{\text {pit2 }}<0.0001$; unpaired Student's $t$-test. (f) Relative transcript levels of $c m u 1$, pep1 and pit2 in cells grown in infected plant tissue at 1-2 d.p.i. Effector expression in infecting control strains was set as $100 \%$. Mean \pm s.e. and sample sizes $n$ (=number of experiments) are shown. ${ }^{\star \star}$ Significance at error probability $P_{\mathrm{cmu} 1}=0.0021$ and $P_{\text {pep } 1}=0.0096 ;{ }^{\star \star \star}$ significance at error probability $P_{\text {pit } 2}<0.0001$; unpaired Student's $t$-test. ( $(\mathbf{g})$ Secretion of $\mathrm{Cmu1-mCherry}$ in control and $\Delta$ hok 1 mutants. Note that secretion of the effector in $\Delta$ hok1 mutants is reduced compared with control strain. Scale bar, $5 \mu$ m.

Shok1 mutants, rab5a null mutants caused only mild pathogenicity symptoms (Fig. 6c,d), and, again, transcription and secretion of all tested effectors were significantly reduced (Fig. 6e-g). We conclude that inhibition of EE motility, either by expression of EAP, deletion of the motor adaptor Hok1 or deletion of $r a b 5 a$, reduces effector transcription and secretion, thereby preventing the fungus from successful colonization of plant tissue.

An endosome-associated MAPK controls effector transcription. Our observations indicated that Rab5a-positive EEs mediate retrograde signaling that initiates transcription and subsequent secretion of effectors. We therefore set out to identify potential signalling components in this pathway. In mammalian cells, purified Rab5-positive EEs contain numerous long-range signalling proteins. Among these are MAPKs of the extracellular signal-regulated kinase (ERK) signalling pathway, including B-Raf (B-rapidly accelerated fibrosarcoma), MEK1 (MAPK or ERK kinase), ERK1/2 (ERKs 1 and 2) and kinase p38 (MAPK p38; overview in ref. 21). In addition, the GTPases Rap1
(Ras-related protein 1), the adaptor protein Gab2 (growth factor receptor-bound protein 2; ref. 18) and the EE-associated adaptor protein, phosphotyrosine interaction, $\mathrm{PH}$ domain and leucine zipper containing 1 (APPL1; ref. 41) are involved in signalling. Finally, components of the Akt signalling pathway, such as glycogen synthase kinase- 3 beta (GSK3 $\beta$; ref. 42) and the transforming growth factor beta (TGF- $\beta$ )/smad signalling pathway, including the signalling mediators SMAD2 and SMAD4 (ref. 43), the FYVE-domain containing endofin ${ }^{44}$ and the SMAD anchor for receptor activation (SARA; ref. 45) transport signals from the plasma membrane to the nucleus (overview in refs 19,21). We used the sequences of these human proteins (see Methods section for accession codes) in a reciprocal BLAST search and screened the published genome sequence of $U$. maydis for fungal orthologues of any of these signalling proteins. We did not find APPL-like proteins or components of the SMAD/SARA pathway nor did we identify homologues of Gab2 or Rap1. However, we found a GSK3 $\beta$ homologue (64\% identical to human GSK3 $\beta$; NCBI accession code: XP_756707.1) and the MAPK Kpp2/Ubc2 (refs 46,47; NCBI accession code: XP_759452.1; 49\% identical to human ERK1 and 54\% identical 
a

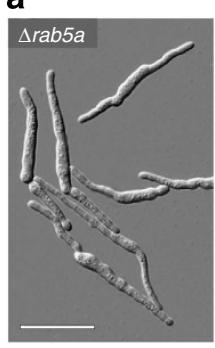

b

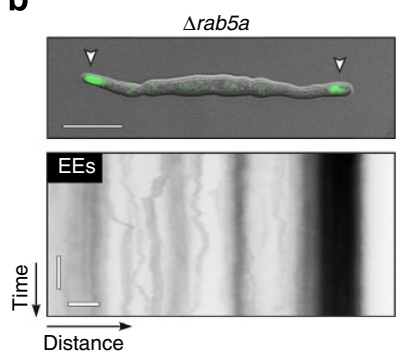

C

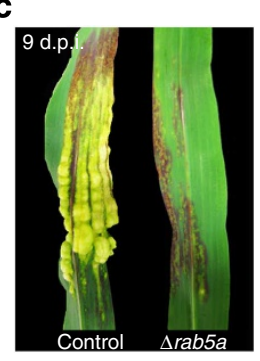

d

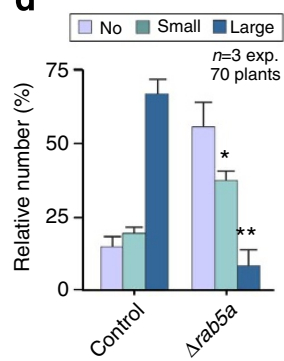

e

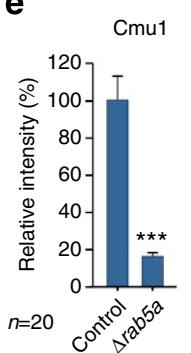

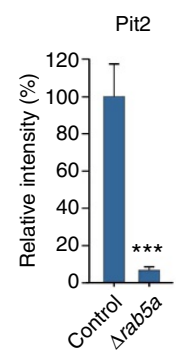

f
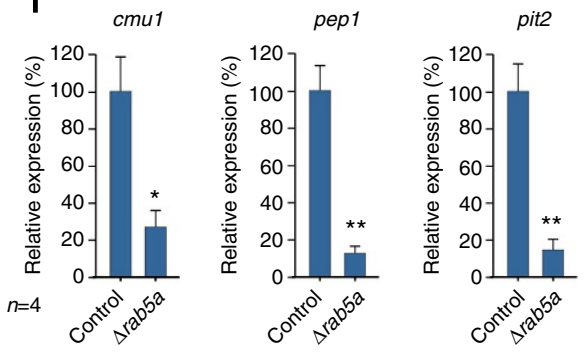

\section{g}
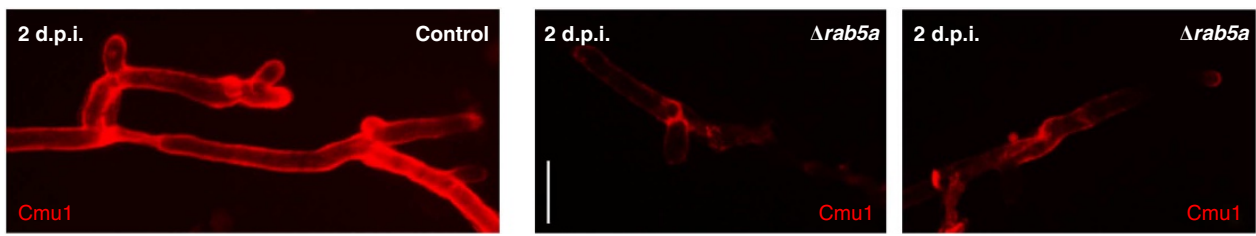

Figure 6 | Effector secretion and pathogenicity are impaired in rab5a null mutants. (a) Phenotype of rab5a deletion mutants. Scale bar, $20 \mu \mathrm{m}$. (b) EE localization and motility in rab5a deletion mutants. Upper panel shows EE clusters (Yup1-GFP, green, arrowheads) at the poles of the short mutant cells. Lower kymograph shows the absence of EE motility in $\Delta$ rab5a mutants. Scale bars, $3 \mu \mathrm{m}$ (upper panel), $5 \mathrm{~s}$ (vertical, lower panel) and $2 \mu \mathrm{m}$ (horizontal, lower panel). (c) Plant disease symptoms at 9 days after infection of maize plants with control and $\Delta$ rab5a strains. (d) Plant symptoms at 14 days after infection with control and rab5a deletion mutants. Mean \pm s.e. and sample sizes $n$ (= number of experiments (exp)) are shown. ${ }^{\star}$ Different from control at error probability $P=0.019$; ${ }^{\star \star}$ different from control at error probability $P=0.0047$; unpaired Student's $t$-test. (e) Secretion of fluorescent effectors indicated by the signal intensity at the periphery of the invasive hyphae in plants at 2 d.p.i. Mean \pm s.e. and sample size $n$ from one experiment is shown. The result was confirmed by a non-quantitative experiment. ${ }^{\star \star \star}$ Significance at error probability $P<0.0001$; unpaired Student's $t$-test. (f) Relative transcript levels of $c m u 1$, pep1 and pit2 in cells grown in infected plant tissue at 1-2 d.p.i. Effector expression in infecting control strains was set as $100 \%$. Mean \pm s.e. and sample sizes $n$ ( $=$ number of experiments) are shown. ${ }^{*}$ Significance at error probability $P_{\mathrm{cmul}}=0.0257$, ${ }^{\star \star}$ significance at error probabilities $P_{\text {pep } 1}=0.0083$ and $P_{\text {pit2 }}=0.007$; unpaired Student's $t$-test. (g) Secretion of Cmu1-mCherry in Control and $\Delta$ rab5a mutants. Note that secretion in $\Delta$ rab5 is reduced, compared to the control strain. Scale bar, $5 \mu \mathrm{m}$.

to human MAPK p38 and ERK2) and the MAPK kinase kinase Kpp4/Ubc4 (refs 48,49; 37\% identical to human MEK1). $U$. maydis contains a single MAPK module ${ }^{49}$. This consists of the MAPKK kinase Kpp4/Ubc4 (refs 48,49), the downstream MAPK Fuz7 (ref. 50) and the MAPKs Kpp2/Ubc2 (refs 46,47), Kpp6 (ref. 51) and Crk1 (refs 52,53 and Supplementary Fig. 5a). All MAPK module components are required for full virulence in $U$. maydis ${ }^{46,47,49-52}$ and thus could be involved in virulenceassociated signalling. We therefore fused single GFP to all these kinases and to the GSK3 $\beta$ homologue, and asked whether any of these show long-range motility within hyphal cells. In these experiments, the MAPK Crk1 showed constant and directed movements. The MAPKK kinase Kpp4 and GSK3 $\beta$ homologue occasionally moved, whereas the MAPK kinase Fuz7 and the MAPKs Kpp2 and Kpp6 were not motile (Supplementary Fig. 5b). Interestingly, all moving kinases were also concentrated in the fungal nucleus (Supplementary Fig. 5c), which supports the notion that they serve roles in membrane-to-nucleus signalling.

In the initial localization experiments, very faint Crk1-GFP signals moved over long distances. To test whether Crk1 is transported via retrograde moving EEs, we fused the endogenous copy of crk1 to a triple-GFP tag and co-expressed the fusion protein with endosomal mCherry-Rab5a in a strain that forms hyphal cells in liquid culture. We observed that Crk1-GFP 3 localized to rapidly moving Rab5a-positive EEs (Fig. 7a and Supplementary Movie 7). Retrograde Crk1-GFP 3 signals travelled for up to $30 \mu \mathrm{m}$ (Fig. 7a), which is consistent with the notion that the MAPK could participate in retrograde signalling to the nucleus during plant infection. To test this idea further, we expressed Crk1-GFP 3 in the solo-pathogenic strain SG200 and investigated Crk1 motility in planta. We found that the MAPK also travelled over long distances in invasive hyphae and localized in the nucleus (Fig. 7b and Supplementary Fig. 6a). This result suggests that Crk1 could participate in transmitting signals from the hyphal tip to the nucleus, thereby controlling effector transcription and secretion. We addressed this possibility by deleting $c r k 1$ in a strain that expressed Cmul-mCherry. Surprisingly, we found that effector secretion was significantly increased in the absence of Crk1 (Fig. 7c, both images are identically processed; Fig. 7d), suggesting an inhibitory signalling function. We therefore set out to test whether expression of $\mathrm{cmul}$ was induced by deletion of the MAPK crk1. The resultant $c r k 1$ 
a

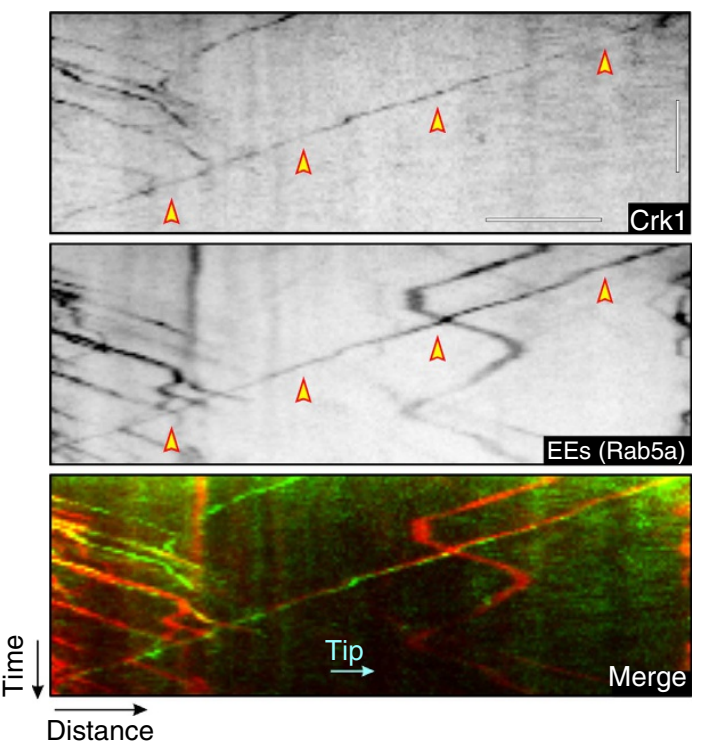

b

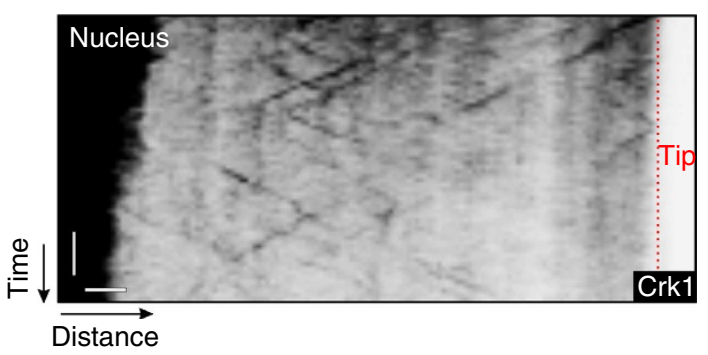

C

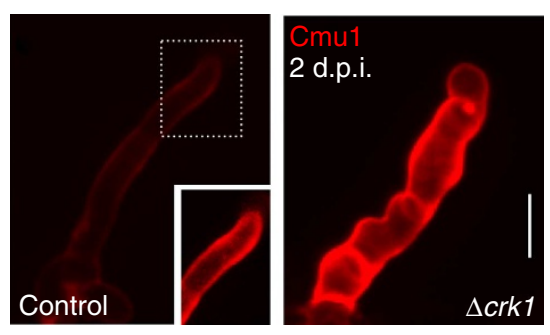

e
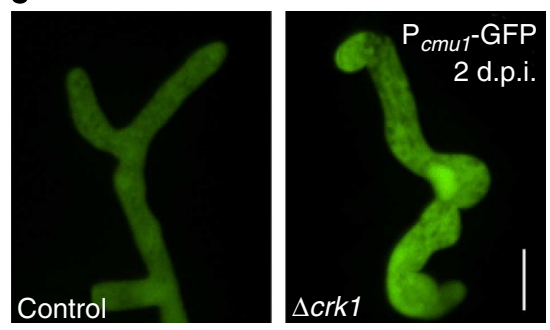

d

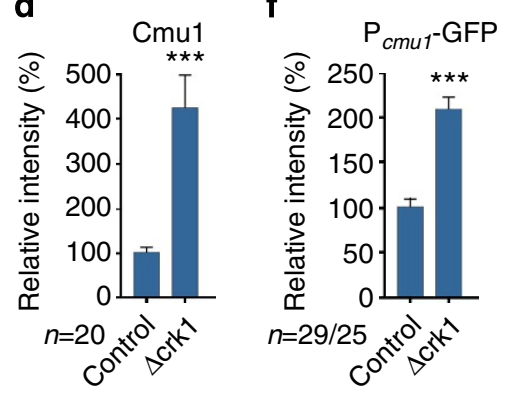

Figure 7 | EE-associated localization of the MAPK Crk1 and its role in effector secretion and transcription. (a) Kymographs showing motility of Crk1-GFP 3 (Crk1) on EEs (Rab5a, arrowheads). Direction towards the hyphal tip is indicated (Tip). Note that the endogenous copy of crk1 was fused to a triple-GFP tag. Motility was observed in a photo-bleached area. The upper two kymographs are contrast inverted. Scale bars, $5 \mathrm{~s}$ (vertical) and $5 \mu \mathrm{m}$ (horizontal). See Supplementary Movie 7. (b) Kymographs showing motility of Crk1-GFP 3 in a fungal hypha during invasion of plant tissue. Note that trajectories of $\mathrm{Crk1}_{-} \mathrm{GFP}_{3}$ signals are discontinuous, as signals move in and out of the confocal focus. Scale bars, $3 \mu \mathrm{m}$ (upper panel), $5 \mathrm{~s}$ (vertical, lower panel) and $2 \mu \mathrm{m}$ (horizontal, lower panel). See Supplementary Movie 8. (c) Secretion of fluorescent Cmu1 effector in control and crk1 null mutants ( $\Delta$ crk1). Both images were scaled identically to show different degree of secreted Cmu1-mCherry. Inset shows Cmu1-mCherry after scaling the control images. Scale bar, $5 \mu \mathrm{m}$. (d) Secretion of fluorescent $\mathrm{Cmu} 1$ indicated by the signal intensity at the periphery of the invasive control and $\Delta c r k 1$ mutant hyphae in plants at 2 d.p.i. Mean \pm s.e. and sample size $n$ from two experiments is shown. ${ }^{\star \star \star}$ Significance at error probability $P=0.0005$; unpaired Student's $t$-test.

(e) Expression of cytoplasmic GFP under the cmu1 promoter in invasive control and $\Delta c r k 1$ mutant hyphae in plants at 2 d.p.i. Both images were scaled identically. Scale bar, $5 \mu \mathrm{m}$. (f) Expression of cytoplasmic GFP under the cmu1 promoter in invasive control and $\Delta c r k 1$ mutant hyphae in plants at 2 d.p.i. Mean \pm s.e. and sample size $n$ from two experiments is shown. ${ }^{\star \star \star}$ Significance at error probability $P<0.0001$; unpaired Student's $t$-test.

null mutants, however, showed a defect in morphology and were significantly impaired in penetration of the plant. Consequently, RT-PCR analysis of cmul could not be reliably carried out because of the small proportion of fungal hyphae that successfully penetrated host tissue, which would normally express cmu1 specifically. To circumvent this problem, we made a transcriptional fusion of cytoplasmic GFP under the control of the cmu1 promoter $\left(P_{\mathrm{cmul}}\right)$ and quantitatively analysed the GFP signal intensity in control hyphae at 1 d.p.i., when the fungus had not yet invaded the plant, and 2 d.p.i., when fungal cells were found inside the plant tissue. We found only traces of cytoplasmic GFP at 1 d.p.i., but a strong fluorescent signal when the fungus had penetrated the plant (Supplementary Fig. 6b,c). This is consistent with the induction of $P_{\text {cmul }}$ during plant invasion. Significantly, less fluorescence was detected in $\Delta$ hok1 mutants (Supplementary Fig. 6b,c), which is consistent with the role of EE motility in effector transcription. We next generated a crk1 null mutant, expressing the $P_{\mathrm{cmu1}}$-GFP construct. We infected plants and visualized infection structures after 2 d.p.i. Interestingly, we found a significant increase in cytoplasmic GFP fluorescence, indicating that $P_{\mathrm{cmu} 1}$ is more strongly induced in $\Delta c r k 1$ mutants (Fig. $7 \mathrm{e}, \mathrm{f}$ ). When considered together, these results provide evidence that the EE-associated MAPK, Crk1, acts as a repressor of effector transcription.

\section{Discussion}

An increasing body of evidence suggests that pathogenic fungi and oomycetes secrete a large repertoire of effector proteins into plant cells to suppress plant immunity and facilitate biotrophic growth $^{1,3-5,8-13,29}$. In this report, we provide evidence that motility of EEs is essential for effector production and subsequent secretion by the filamentous fungal pathogen $U$. maydis. This conclusion is supported by several independent experimental strategies. First, we generated an artificial anchor protein EAP that consists of a mutated kinesin-1 motor head that tightly binds 
to microtubules ${ }^{31}$ and the C-terminal Phox domain of the SNARE Yup1 (ref. 23). Phox domains bind to the lipid PtdIns3P (ref. 54), which is, itself, enriched in the membrane of $\mathrm{EEs}^{55}$. Strong expression of the chimaeric EAP almost abolished EE motility and significantly reduced the secretion of three tested effector proteins into the apoplastic space between the fungus and the plant cell. One could argue that the presence of EAP simply blocks microtubule-dependent delivery of effector proteins. We consider this possibility unlikely, however, because microtubulebased membrane trafficking in U. maydis depends on kinesin-1, kinesin-3 and dynein ${ }^{30}$, and EAP expression did not impair motility of fluorescent kinesin-3 or dynein nor did it affect delivery of chitin synthase Mcs1 to the growing tip of the cell, a process depending on kinesin-1 (ref. 33). We conclude from these results that EAP specifically blocks EE movement but does not affect other membrane trafficking pathways. Consequently, we consider it very likely that impaired effector secretion is a consequence of reduced EE motility. This notion is further supported by the observation that hok1 and rab5a null mutants also show almost identical defects in effector secretion. It was recently reported that hok1 null mutants have a defect in motorto-cargo coupling, which results in a severe reduction in EE transport ${ }^{37}$. A similar defect is seen in rab5a null mutants, although these cells are most likely impaired in various EE functions, because this GTPase is of central importance for motility, identity and function of these organelles ${ }^{40}$. However, when taken together, all three mutant lines (EAP-expressing, hok 1 and rab5a null mutants) show very significant reductions in secretion of the three tested effectors. Motile EEs therefore appear to be essential during the interaction of the fungal pathogen and the plant cell.

How then do moving EEs support effector secretion during plant infection? We recognized that elongated fungal cells face a considerable challenge during host infection in communicating between the invading distal tip and the nucleus. This is clearly necessary in order to produce effector proteins specifically during the very early stages of infection. In $U$. maydis, the nucleus is, for instance, $\sim 22 \mu \mathrm{m}$ behind the invading hyphal tip. In the crowded cytoplasm, even a few micrometres pose an insurmountable problem because passive diffusion is severely hindered ${ }^{56}$. This suggests that communication between the hyphal tip and nucleus involves an active transport-dependent signalling mechanism. In mammalian neurons, such long-range signalling involves EEs, which travel along microtubules, delivering signalling components from the plasma membrane to the nucleus ${ }^{19-21}$. Long-range and microtubule-dependent motility of EEs have been reported in $U$. maydis ${ }^{23,26}$ and other fungi ${ }^{24,25}$ (overview in ref. 22), and we have previously speculated that moving fungal EEs might participate in retrograde signalling ${ }^{57}$. The evidence provided here is consistent with such a function. The observed defects in effector secretion, for example, are most likely owing to impaired effector gene transcription as a consequence of blocked EE-dependent retrograde signalling (Fig. 8). Thus, EE motility acts at the level of transcription, which implies a novel, endosome-based signalling mechanism that mediates communication between the fungal nucleus and invading hyphal tip, and which is essential for fungal pathogenicity.

A role for fungal EEs in long-range signalling has not hitherto been described. Indeed, our understanding of the signalling pathways associated with fungal EEs is in its infancy. In mammalian cells, several signalling pathways are located on EEs that include the MAPKs of the ERK signalling pathway ${ }^{19,21}$. Our search for homologues of retrograde signalling components, associated with EEs in humans, revealed known components of a MAPK module. This included the MAPK Kpp2/Ubc2, which is shown to be involved in pathogenic development ${ }^{46,47}$, and
Invasion site selection

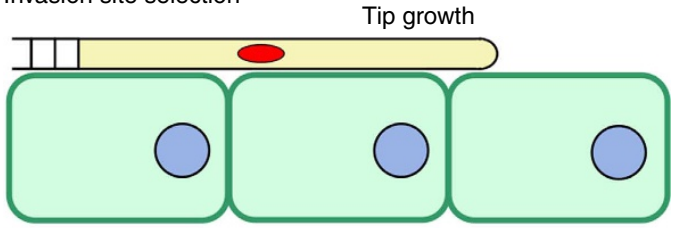

Endocytosis-dependent plant recognition

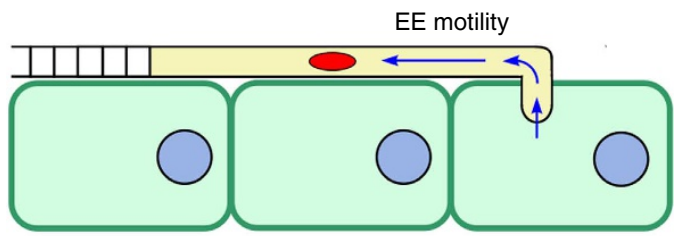

Effector production and secretion

Effector transcription

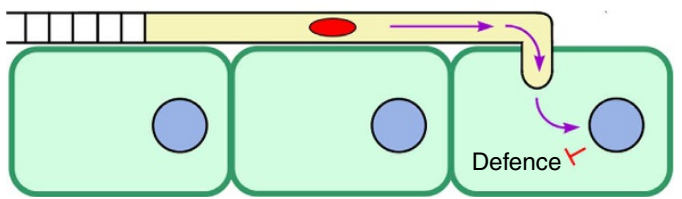

Figure 8 | Model for retrograde EE motility in effector-mediated plant invasion by pathogenic fungi. The invading hyphal tip perceives plant cues during the initial stages of infection. Endocytic uptake relays this information to motile EEs that transport the signal to the nucleus. This retrograde signalling mechanism triggers effector gene expression. Subsequently, effectors are secreted at the hyphal tip to suppress plant immunity and facilitate fungal invasion.

therefore is a good candidate for EE-associated signalling. However, Kpp2 did not show directed movement, whereas the MAPK Crk1 was transported by EEs over long distances. This observation, coupled with localization of Crk1 in the fungal nucleus, and de-repressed effector transcription and secretion, strongly suggests that Crk1 participates in long-range EE-based signalling. We also find the MAPK kinase Fuz7 and the MAPKK kinase Kpp4 in the nucleus, and at least Kpp4 showed occasional motility. Therefore, we consider it most likely that the MAPK module in U. maydis delivers signals from the plasma membrane into the nucleus. Crk1 appears to be of key importance in this pathway, which adds to the known roles of Crk1 in morphogenesis ${ }^{52}$ and mating ${ }^{53}$.

Surprisingly, crk1 null mutants showed increased expression and secretion of effector protein. This suggests that Crk1 is a negative regulator that represses effector transcription. We speculate that it might therefore counteract an unknown EEbound phosphatase to collectively fine-tune expression of effector genes during infection. Such balanced activities of phosphatases and kinases mediate many essential cellular processes, including cell-cycle progression ${ }^{58}$, axonal migration and transport ${ }^{59,60}$, and various signalling pathways ${ }^{61}$. Finally, it is worth mentioning that Gsk $3 \beta$ also showed directed movement in the cell. Mammalian Gsk3 $\beta$ is a direct target of the serine/threonine kinase Akt (ref. 62), and both dynamically interact with the Rab5-effector APPL1 on $\mathrm{EEs}^{42}$. U. maydis does not contain an APPL homologue, and whether the Gsk $3 \beta$ homologue is associated with fungal EEs is not yet clear. However, the identification of an EE-associated and MAPK-based signalling pathway in effector production raises the possibility that EEs have more widespread roles in the plasma membrane-to-nucleus signalling than has hitherto been recognized in pathogenic fungi. 


\section{Methods}

Strains and plasmids. All $U$. maydis strains were constructed in the genetic background of AB33 (ref. 63) or SG200 (ref. 35; Table 1). To observe EEs motility during plant infection, plasmid poGRab5a (ref. 64) was integrated ectopically into strain SG200, resulting in SG200GRab5a. Strains AB33mChRab5a and AB33Kin3G_mChRab5a were generated by integrating the plasmid $\mathrm{po}_{\mathrm{m}} \mathrm{ChRab5a}$

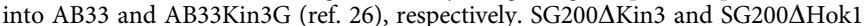
were generated by deleting the endogenous kin 3 or hok1 gene using $\mathrm{p} \Delta \mathrm{Kin} 3$ (ref. 27) or $\mathrm{p}^{\mathrm{H}} \Delta$ Hok1 (ref. 37), respectively. SG200Cmu1Ch_ $\Delta$ Hok1, SG200Pep1Ch_AHok1 and SG200nPit2Ch_ $\Delta$ Hok1 were generated by deleting the endogenous hok1 gene using $\mathrm{p}^{\mathrm{N}} \Delta$ Hok1 (ref. 37). Deletions were confirmed by Southern blot. Ectopic integration of plasmid pCoGRab5a (ref. 65) resulted in SG200AKin3_GRab5a. All plasmids were generated by standard cloning procedures or in vivo recombination in the yeast Saccharomyces cerevisiae ${ }^{66}$ Detailed cloning information is provided in the Supplementary Methods.

Growth conditions. All cultures of $U$. maydis strains were grown overnight at $28{ }^{\circ} \mathrm{C}$ in either $\mathrm{YEPS}_{\text {light }}$ medium ( $1.0 \%$ yeast extract, $0.4 \%$ peptone and $0.4 \%$ sucrose) or complete medium (CM), containing $1 \%(\mathrm{w} / \mathrm{v})$ glucose $\left(\mathrm{CM}_{\mathrm{glc}}\right)$ or $1 \%(\mathrm{w} / \mathrm{v})$ arabinose $\left(\mathrm{CM}_{\text {ara }}\right)$ at 200 r.p.m. Hyphal growth was induced by transferring cells, grown in $\mathrm{CM}_{\mathrm{glc}}$, into nitrate minimal (NM) medium supplemented with $1 \%(\mathrm{w} / \mathrm{v})$ glucose. Cells were grown under these conditions for $5-10 \mathrm{~h}$ at 200 r.p.m. $\left(28^{\circ} \mathrm{C}\right)$.

Microscopic techniques. Spinning disc confocal microscopy of infected plant leaves was performed using a VisiScope Confocal Cell Explorer (Visitron System, Munich, Germany) that consisted of an IX81 motorized inverted microscope (Olympus, Hamburg, Germany), a CSU-X1 Spinning Disc unit (Yokogawa, Tokyo, Japan), a PlanApo UPlanSApo $\times 63 / 1.35$ oil objective (Olympus, Hamburg, Germany) and a Photometrics CoolSNAP HQ2 camera (Roper Scientific, Germany). The fluorescent tags and dyes were excited using a VS-LMS6 Laser Merge System with two solid-state lasers $(488 \mathrm{~nm} / 100 \mathrm{~mW}$ and $561 \mathrm{~nm} / 100 \mathrm{~mW}$, Visitron System) and a $405-\mathrm{nm} / 100-\mathrm{mW}$ diode laser, which was 50/50 split. For photo-activation studies, the $405-\mathrm{nm} / 100-\mathrm{mW}$ diode laser split and directed into a 2D-VisiFRAP Realtime Scanner (Visitron System). Co-observation of GFP and RFP was done using an OptoSplit II LS Image Splitter (Cairn Research Limited, Faversham, UK). All parts of the system were under the control of the software package VisiView (Visitron System)

Laser-based epi-fluorescence microscopy of cultured cells was done as previously described ${ }^{27}$. In brief, cells were placed onto a $2 \%(\mathrm{w} / \mathrm{v})$ agar cushion and immediately observed using an IX81 motorized inverted microscope, equipped with a PlanApo $\times 100 / 1.45$ Oil TIRF (Olympus, Hamburg, Germany). A VS-LMS4 Laser-Merge-System with solid-state lasers $(488 \mathrm{~nm} / 50 \mathrm{~mW}$ or $75 \mathrm{~mW}$ and $561 \mathrm{~nm} / 50 \mathrm{~mW}$ or $75 \mathrm{~mW}$; Visitron System) was used to excite the fluorescent protein tags. Photo-bleaching experiments were done using a $405-\mathrm{nm} / 60-\mathrm{mW}$ diode laser, which was dimmed by an ND 0.6 filter, resulting in $15 \mathrm{~mW}$ output power, coupled into the light path by an OSI-IX 71 adaptor (Visitron System). The system was regulated by a UGA-40 controller (Rapp OptoElectronic GmbH, Hamburg, Germany) and a VisiFRAP 2D FRAP control software for MetaSeries 7.5.1 (Visitron System). Dual imaging of GFP and RFP was carried out using a Dual-View Micro imager (Photometrics, Tucson, USA) equipped with a dual-line beam splitter (z491/561, Chroma, Rockingham, USA) with an emission beam splitter (565 DCXR, Chroma, Rockingham, USA), an ET-Bandpass 525/50 (Chroma, Rockingham, USA) and a BrightLine HC 617/73 (Semrock, Rochester, USA). A Photometrics CoolSNAP HQ2 camera was used to capture all images. All parts of the system were under the control of the software package MetaMorph (MDS Analytical Technologies, Winnersh, UK)

Scanning electron microscopy was performed using yeast-like cells of strain SG200 $\Delta$ Hok1, which grown on CM agar supplemented with $1 \%$ glucose and $1 \%$ charcoal for 5 days at $28^{\circ} \mathrm{C}$. Images were acquired using Nikon D5000 camera that was connected to the Nikon stereomicroscope SMZ800 (Nikon, Kingston, Surrey, UK). The $10-\mathrm{mm}$ diameter agarose discs containing yeast-like and hyphal cells were attached to a cryostage and rapidly frozen in liquid nitrogen slush, followed by water sublimation at $-95^{\circ} \mathrm{C}$ for $3 \mathrm{~min}$ in Jeol JSM-6390LV scanning electron microscope (JEOL, Ltd, Welwyn Garden City, UK). This was followed by gold sputter coating using an Alto 2100 chamber (Gatan Ltd., Oxfordshire, UK) and observation in a Jeol JSM-6390LV scanning electron microscope (JEOL, Ltd).

\section{Quantitative analysis of motility ex planta. EE motility in the presence or} absence of EAP was measured in cells grown in $\mathrm{NM}_{\text {glu }}$ (no EAP) and $\mathrm{NM}_{\text {ara }}$ ( + EAP, expression induced for $5 \mathrm{~h}$ ). GFP-Rab5a signals that did not move for $10 \mathrm{~s}$ were considered as non-motile EEs. The effect of EAP on flux of fluorescent dynein was measured within photo-bleached hyphal cells, beginning $5 \mu \mathrm{m}$ behind the tip and extending for $20 \mu \mathrm{m}$ in $\mathrm{NM}_{\text {glu }}(15 \mathrm{~h})$ and $\mathrm{NM}_{\text {ara }}$ (expression induced for $15 \mathrm{~h}$ ). Motility was analysed in kymographs. Kinesin-3 motility was measured in photobleaching apical parts of yeast-like and hyphal cells, using a $405-\mathrm{nm} / 60-\mathrm{mW}$ diode laser, dimmed to $15 \mathrm{~mW}$ output using kymographs. Only signals that did not co-localize with mCherry-Rab5a were counted and compared with all kinesin-3GFP signals, irrespective of co-localization with EEs. The degree of secretion of
Mcs1-GFP ${ }_{3}$ was estimated by measuring the average intensity of the GFP signal in the apical parts of cells forming a medium-sized bud. The intensity values were corrected by the cytoplasmic background.

Fungal plant infection. U. maydis strains were grown in either $\mathrm{YEPS}_{\text {light }}, \mathrm{CM}_{\text {glc }}$ or $\mathrm{CM}_{\text {ara }}$ to an $\mathrm{OD}_{600}$ of $0.8-1.0$. Cells were harvested by centrifugation and resuspended in water. A quantity of 0.2 or $0.5 \mathrm{ml}$ of this cell suspension $\left(\mathrm{OD}_{600} \sim 1.0\right)$ were injected into 10-day-old maize plants (Golden Bantam; Chase Organics Ltd., Hersham, Surrey, UK). Pathogenicity assays were performed as described previously ${ }^{67}$. In brief, infected plants were grown in a GroBank (CSF Plant Climatics, Wertingen, Germany; $16 \mathrm{~h}$ light, $8 \mathrm{~h}$ darkness, $28^{\circ} \mathrm{C}$ ). Eight- to nine-day-old plants were infected by injection of $0.5 \mathrm{ml} \mathrm{U}$. maydis cells, pre-grown to $\mathrm{OD}_{600}=0.8$ and washed with water into the stem of the plant. Disease symptoms were scored at 9-14 days after infection. The scoring criteria were as follows: 'no tumours' (chlorosis and antocyanin formation), 'small tumours' (chlorosis, antocyanin formation and tumours up to $3 \mathrm{~mm}$ ) and 'large tumours' (chlorosis, antocyanin formation and tumours over $3 \mathrm{~mm}$ ). All infection assays were done in at least three experiments and compared by Student's $t$-test by using the software Prism 4.03 (GraphPad Software Inc., La Jolla, CA, USA).

Microscopy of infected plant tissue. Propidium iodide/wheat germ agglutininAF488 and diaminobenzidine (Sigma-Aldrich, UK) staining in infected plant leaves was done as described previously ${ }^{67}$. In brief, infected leaf sections were incubated in staining solution $\left(1 \mathrm{mg} \mathrm{ml}^{-1}\right.$ diaminobenzidine, $0.05 \%(\mathrm{v} / \mathrm{v})$ Tween 20 and $10 \mathrm{mM} \mathrm{Na}_{2} \mathrm{HPO}_{4}$ ) under mild vacuum for $60 \mathrm{~min}$, followed by $4 \mathrm{~h}$ shaking at 80-100 r.p.m. at room temperature. Samples were incubated in ethanol:acetic acid:glycerol $(3: 1: 1)$ and stored in fresh de-stain solution at $4{ }^{\circ} \mathrm{C}$ (at $90^{\circ} \mathrm{C}$ for $15 \mathrm{~min}$ ). Before microscopy, samples were washed in PBS, $\mathrm{pH}$ 7.4, and microscopically analysed.

To visualize nucleus and effector secretion in early stages of infection, plants were infected with the strain SG200G ${ }_{3} \mathrm{NLSR}_{3}$ and SG200CmulCh_G experimental usage of strains, see Supplementary Table 2 ). An $\sim 1 \times 1-\mathrm{cm}$ region at $\sim 5 \mathrm{~cm}$ beneath the infection point was taken from the third inner leaf and placed on Carolina observation Gel (Carolina Biological Supply Company, Burlington, USA). The invasion site was identified by bright-field microscopy, followed by acquiring Z-stacks capturing red and green fluorescence, using an OptoSplit II LS Image Splitter. Z-stacks covered $30 \mu \mathrm{m}$ at $0.2 \mu \mathrm{m}$ step size (exposure time of $200 \mathrm{~ms}$, binning 1 and the $488-/ 561-\mathrm{nm}$ laser at 20\%/50\%). After merging stacks, maximum projections and three-dimensional reconstructions were done using MetaMorph (Molecular Devices, Winnersh, UK).

EE motility inside invading fungal cells was measured after infection of maize plants with strain SG200GRab5a. Leaf samples were collected at several time points post infection, and GFP-Rab5a motility was recorded in randomly selected invasive hyphal cells. The proportion of moving EEs was determined in movies, covering $8-10 \mathrm{~s}$ observation time.

To analyse EE motility in fungal cells at 1 d.p.i., infected leaf tissue was prepared for microscopic analysis. The samples were incubated in $2 \mu \mathrm{g} \mathrm{ml}^{-1}$ Calcofluor solution (F3543-Fluorescent Brightener 28, Sigma, UK) for $30 \mathrm{~s}$, water rinsed and observed on Carolina Observation Gel, using a spinning disc confocal microscope. Invasion sites were identified and Z-stacks were generated as described above. Calcofluor was excited using a $405-\mathrm{nm}$ laser at $15 \%$ output power. In photoactivation experiments, paGFP-Rab5a was excited at the invasion site using a focussed $405-\mathrm{nm}$ laser at $15 \%$ output power, followed by immediate acquisition of 150 planes using the OptoSplit II LS Image Splitter (Cairn Research Limited), and the 488 - and $561-\mathrm{nm}$ observation lasers at $30 \%$ and $50 \%$ output power, respectively. Motility of photo-activated EEs was analysed in kymographs by using MetaMorph.

To test for $\operatorname{crg} 1$ or mig1 promoters activities in planta, maize plants were infected with $0.5 \mathrm{ml}$ suspension of strain SG200 $\mathrm{crg}$ G and SG200migG, and pregrown in $\mathrm{CM}_{\text {ara }}$ or $\mathrm{CM}_{\mathrm{glc}}$. Maize tissue samples were collected and observed using an IX81 microscope and a VS-LMS4 Laser Merge System, the 488-nm observation laser at $100 \%$ output power at an exposure time of $150 \mathrm{~ms}$ and image binning 1 .

Quantitative analysis of fluorescent intensity of Cmu1-mCherry, Pep1-mCherry and Pit2-mCherry was done by measuring the average fluorescent intensity at the periphery of hyphal cells of strains SG200GRab5a_cEAP_Cmu1Ch, SG200GRab5a_cEAP_Pep1Ch and SG200GRab5a_cEAP_APit2_nPit2Ch that showed reduced or no EE motility. Images were taken at $200 \mathrm{~ms}$ exposure time. The average fluorescence intensity over length of $\sim 5 \mu \mathrm{m}$ was measured and corrected for the background intensity in the cytoplasm. All statistic testing was done using the software Prism 4.03 (GraphPad Software Inc.).

To analyse expression of cytoplasmic GFP expressed under the $\mathrm{cmul}$ promoter images (200 ms exposure time, spinning disc microscope) were taken of strains

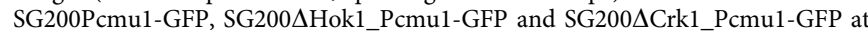
1 and 2 d.p.i. The average fluorescent intensity was measured and corrected for the background adjacent to the cell. All statistic testing was done using the software Prism 4.03 (GraphPad Software Inc.).

Quantitative RT-PCR analysis. Quantitative analysis of effector expression was performed using qRT-PCR, according to published protocols ${ }^{9,11,13,68}$. In brief, leaf 
samples were removed 2-4 $\mathrm{cm}$ beneath the injection point and harvested from four independent experiments, 30 plants each. The material was frozen in liquid nitrogen, ground by mortar and pestle, and total RNA was extracted by using an RNeasy Plant Mini Kit (Qiagen, Hilden, Germany) with DNase I treatment, according to the manufacturer's instructions. cDNA was synthesized using SuperScript III First-Strand Synthesis SuperMix (Invitrogen, Carlsbad, USA). qRTPCR was performed with an Mx3005P thermocycler (Stratagene, La Jolla, USA) by using Brilliant III Ultra-Fast SYBR Green QPCR Master Mix (Stratagene) and $\sim 250$ or $500 \mathrm{ng}$ cDNAs, estimated by NanoDrop ND-1000 (LabTech International Ltd, East Sussex, UK), from samples of liquid cultures or plants, respectively. qRTPCR conditions were as follows: $3 \mathrm{~min}$ at $95^{\circ} \mathrm{C}$, followed by 40 cycles of $95^{\circ} \mathrm{C}$ for $20 \mathrm{~s}$ and $60^{\circ} \mathrm{C}$ for $20 \mathrm{~s}$. To normalize expression levels, the constitutively expressed peptidyl-prolyl cis-trans isomerase gene ( $p p i)$ was used, as described previously ${ }^{9}$.

Bioinformatics. Putative components of EE-associated signalling were identified by reciprocal BLAST search (http://blast.ncbi.nlm.nih.gov/Blast.cgi). All protein sequences used were fetched from the NCBI server (http://www.ncbi.nlm.nih.gov/ pubmed). Accession codes of the protein sequences used in this approach were as follows: human ERK1: P27361.4; human ERK2: P28482.3; human MEK1: NP_005912.1; human MAPK p38: NP_002736.3; human Gab2: BAA76737.1; human RAP1: ABA64473.1; human GSK3beta: NP 002084.2; mouse RAF-B: NP_647455.3; human APPL1: NP_036228.1; human APPL2: NP_001238833.1; human SMAD2: AAC39657.1; human SMAD4: Q13485.1; human endofin: AAL30772.1 and human SARA: NP_004790.2.

\section{References}

1. Dodds, P. N. \& Rathjen, J. P. Plant immunity: towards an integrated view of plant-pathogen interactions. Nat. Rev. Genet. 11, 539-548 (2010).

2. Jones, J. D. \& Dangl, J. L. The plant immune system. Nature 444, 323-329 (2006).

3. Kale, S. D. \& Tyler, B. M. Entry of oomycete and fungal effectors into plant and animal host cells. Cell. Microbiol. 13, 1839-1848 (2011).

4. Valent, B. \& Khang, C. H. Recent advances in rice blast effector research. Curr. Opin. Plant Biol. 13, 434-441 (2010).

5. Rafiqi, M., Ellis, J. G., Ludowici, V. A., Hardham, A. R. \& Dodds, P. N. Challenges and progress towards understanding the role of effectors in plantfungal interactions. Curr. Opin. Plant Biol. 15, 477-482 (2012).

6. Brefort, T. et al. Ustilago maydis as a pathogen. Annu. Rev. Phytopathol. 47, 423-445 (2009).

7. Mendoza-Mendoza, A. et al. Physical-chemical plant-derived signals induce differentiation in Ustilago maydis. Mol. Microbiol. 71, 895-911 (2009).

8. Doehlemann, G. et al. Reprogramming a maize plant: transcriptional and metabolic changes induced by the fungal biotroph Ustilago maydis. Plant J. 56, 181-195 (2008).

9. Doehlemann, G. et al. Pep1, a secreted effector protein of Ustilago maydis, is required for successful invasion of plant cells. PLoS Pathog. 5, e1000290 (2009).

10. Hemetsberger, C., Herrberger, C., Zechmann, B., Hillmer, M. \& Doehlemann, G. The Ustilago maydis effector Pep1 suppresses plant immunity by inhibition of host peroxidase activity. PLoS Pathog. 8, el002684 (2012).

11. Doehlemann, G., Reissmann, S., Assmann, D., Fleckenstein, M. \& Kahmann, R. Two linked genes encoding a secreted effector and a membrane protein are essential for Ustilago maydis-induced tumour formation. Mol. Microbiol. 81, 751-766 (2011).

12. Mueller, A. N., Ziemann, S., Treitschke, S., Assmann, D. \& Doehlemann, G. Compatibility in the Ustilago maydis-maize interaction requires inhibition of host cysteine proteases by the fungal effector Pit2. PLoS Pathog. 9, e1003177 (2013).

13. Djamei, A. et al. Metabolic priming by a secreted fungal effector. Nature 478, 395-398 (2011)

14. Grand, C., Sarni, F. \& Lamb, C. J. Rapid induction by fungal elicitor of the synthesis of cinnamyl-alcohol dehydrogenase, a specific enzyme of lignin synthesis. Eur. J. Biochem. 169, 73-77 (1987).

15. Lamb, C. J., Lawton, M. A., Dron, M. \& Dixon, R. A. Signals and transduction mechanisms for activation of plant defenses against microbial attack. Cell 56, 215-224 (1989).

16. Apostol, I., Heinstein, P. F. \& Low, P. S. Rapid Stimulation of an oxidative burst during elicitation of cultured plant cells: role in defense and signal transduction. Plant Physiol. 90, 109-116 (1989).

17. Flor-Parra, I., Vranes, M., Kamper, J. \& Perez-Martin, J. Biz1, a zinc finger protein required for plant invasion by Ustilago maydis, regulates the levels of a mitotic cyclin. Plant Cell 18, 2369-2387.

18. Delcroix, J. D. et al. NGF signaling in sensory neurons: evidence that early endosomes carry NGF retrograde signals. Neuron 39, 69-84 (2003).

19. Scita, G. \& Di Fiore, P. P. The endocytic matrix. Nature 463, 464-473 (2010).

20. Gould, G. W. \& Lippincott-Schwartz, J. New roles for endosomes: from vesicular carriers to multi-purpose platforms. Nat. Rev. Mol. Cell Biol. 10, 287-292 (2009)
21. Miaczynska, M., Pelkmans, L. \& Zerial, M. Not just a sink: endosomes in control of signal transduction. Curr. Opin. Cell. Biol. 16, 400-406 (2004).

22. Steinberg, G. Endocytosis and early endosome motility in filamentous fungi. Curr. Opin. Microbiol. 20C, 10-18 (2014).

23. Wedlich-Söldner, R., Bölker, M., Kahmann, R. \& Steinberg, G. A putative endosomal t-SNARE links exo- and endocytosis in the phytopathogenic fungus Ustilago maydis. EMBO J. 19, 1974-1986 (2000).

24. Abenza, J. F., Pantazopoulou, A., Rodriguez, J. M., Galindo, A. \& Penalva, M. A. Long-distance movement of Aspergillus nidulans early endosomes on microtubule tracks. Traffic 10, 57-75 (2009).

25. Seidel, C., Moreno-Velasquez, S. D., Riquelme, M. \& Fischer, R. Neurospora crassa NKIN2, a kinesin-3 motor, transports early endosomes and is required for polarized growth. Eukaryot. Cell 12, 1020-1032 (2013).

26. Schuster, M. et al. Kinesin-3 and dynein cooperate in long-range retrograde endosome motility along a nonuniform microtubule array. Mol. Biol. Cell 22, 3645-3657 (2011).

27. Schuster, M., Lipowsky, R., Assmann, M. A., Lenz, P. \& Steinberg, G. Transient binding of dynein controls bidirectional long-range motility of early endosomes. Proc. Natl Acad. Sci. USA 108, 3618-3623 (2011).

28. Kämper, J. et al. Insights from the genome of the biotrophic fungal plant pathogen Ustilago maydis. Nature 444, 97-101 (2006).

29. Tanaka, S. et al. A secreted Ustilago maydis effector promotes virulence by targeting anthocyanin biosynthesis in maize. Elife 3, e01355 (2014).

30. Schuchardt, I., Assmann, D., Thines, E., Schuberth, C. \& Steinberg, G. Myosin-V, Kinesin-1, and Kinesin-3 cooperate in hyphal growth of the fungus Ustilago maydis. Mol. Biol. Cell 16, 5191-5201 (2005).

31. Straube, A., Hause, G., Fink, G. \& Steinberg, G. Conventional kinesin mediates microtubule-microtubule interactions in vivo. Mol. Biol. Cell 17, 907-916 (2006).

32. Bottin, A., Kämper, J. \& Kahmann, R. Isolation of a carbon source-regulated gene from Ustilago maydis. Mol. Gen. Genet. 253, 342-352 (1996).

33. Schuster, M. et al. Myosin-5, kinesin-1 and myosin-17 cooperate in secretion of fungal chitin synthase. $E M B O$ J. 31, 214-227 (2012).

34. Basse, C. W., Stumpferl, S. \& Kahmann, R. Characterization of a Ustilago maydis gene specifically induced during the biotrophic phase: evidence for negative as well as positive regulation. Mol. Cell. Biol. 20, 329-339 (2000).

35. Bölker, M., Genin, S., Lehmler, C. \& Kahmann, R. Genetic regulation of mating and dimorphism in Ustilago maydis. Can. J. Bot. 73, 320-325 (1995).

36. Thordal-Christensen, H., Zhang, Z., Wei, Y. \& Collinge, D. B. Subcellular localization of $\mathrm{H}_{2} \mathrm{O}_{2}$ in plants. $\mathrm{H}_{2} \mathrm{O}_{2}$ accumulation in papillae and hypersensitive response during the barley-powdery mildew interaction. Plant $J$. 11, 1187-1194 (1997).

37. Bielska, E. et al. Hook is an adapter that coordinates kinesin-3 and dynein cargo-attachment on early endosomes. J. Cell. Biol. 204, 989-1007 (2014).

38. Zhang, J., Qiu, R., Arst, Jr H. N., Peñalva, M. A. \& Xiang, X. HookA is a novel dynein-early endosome linker critical for cargo movement in vivo. J. Cell. Biol. 204, 1009-1026 (2014)

39. Fuchs, U., Hause, G., Schuchardt, I. \& Steinberg, G. Endocytosis is essential for pathogenic development in the corn smut fungus Ustilago maydis. Plant Cell 18, 2066-2081 (2006).

40. Zerial, M. \& McBride, H. Rab proteins as membrane organizers. Nat. Rev. Mol. Cell Biol. 2, 107-117 (2001).

41. Miaczynska, M. et al. APPL proteins link Rab5 to nuclear signal transduction via an endosomal compartment. Cell 116, 445-456 (2004).

42. Schenck, A. et al. The endosomal protein Appll mediates Akt substrate specificity and cell survival in vertebrate development. Cell 133, 486-497 (2008).

43. Zhang, Y., Musci, T. \& Derynck, R. The tumor suppressor Smad4/DPC 4as a central mediator of Smad function. Curr. Biol. 7, 270-276 (1997).

44. Chen, Y. G., Wang, Z., Ma, J., Zhang, L. \& Lu, Z. Endofin, a FYVE domain protein, interacts with Smad4 and facilitates transforming growth factor-beta signaling. J. Biol. Chem. 282, 9688-9695 (2007).

45. Tsukazaki, T., Chiang, T. A., Davison, A. F., Attisano, L. \& Wrana, J. L. SARA, a FYVE domain protein that recruits Smad2 to the TGFbeta receptor. Cell 95, 779-791 (1998).

46. Müller, P., Aichinger, C., Feldbrügge, M. \& Kahmann, R. The MAP kinase kpp2 regulates mating and pathogenic development in Ustilago maydis. Mol. Microbiol. 34, 1007-1017 (1999).

47. Mayorga, M. E. \& Gold, S. E. The $u b c 2$ gene of Ustilago maydis encodes a putative novel adaptor protein required for filamentous growth, pheromone response and virulence. Mol. Microbiol. 41, 1365-1379 (2001).

48. Andrews, D. L., Egan, J. D., Mayorga, M. E. \& Gold, S. E. The Ustilago maydis $u b c 4$ and $u b c 5$ genes encode members of a MAP kinase cascade required for filamentous growth. Mol. Plant Microbe Interact. 13, 781-786 (2000).

49. Müller, P., Weinzierl, G., Brachmann, A., Feldbrügge, M. \& Kahmann, R. Mating and pathogenic development of the smut fungus Ustilago maydis are regulated by one mitogen-activated protein kinase cascade. Eukaryot. Cell 2, 1187-1199 (2003). 
50. Banuett, F. \& Herskowitz, I. Identification of fuz7, a Ustilago maydis MEK/MAPKK homolog required for $a$-locus-dependent and -independent steps in the fungal life cycle. Genes Dev. 15, 1367-1378 (1994).

51. Brachmann, A., Schirawski, J., Müller, P. \& Kahmann, R. An unusual MAP kinase is required for efficient penetration of the plant surface by Ustilago maydis. EMBO J. 22, 2199-2210 (2003).

52. Garrido, E. \& Perez-Martin, J. The $c r k 1$ gene encodes an Ime2-related protein that is required for morphogenesis in the plant pathogen Ustilago maydis. Mol. Microbiol. 47, 729-743 (2003).

53. Garrido, E. et al. The induction of sexual development and virulence in the smut fungus Ustilago maydis depends on Crk1, a novel MAPK protein. Genes Dev. 18, 3117-3130 (2004).

54. Lemmon, M. A. Phosphoinositide recognition domains. Traffic. 4, 201-213 (2003).

55. Gillooly, D. J. et al. Localization of phosphatidylinositol 3-phosphate in yeast and mammalian cells. EMBO J. 19, 4577-4588 (2000).

56. Luby-Phelps, K. Cytoarchitecture and physical properties of cytoplasm: volume, viscosity, diffusion, intracellular surface area. Int. Rev. Cytol. 192, 189-221 (2000).

57. Steinberg, G. On the move: endosomes in fungal growth and pathogenicity. Nat. Rev. Microbiol. 5, 309-316 (2007).

58. Kraft, C. Mitotic entry: tipping the balance. Curr. Biol. 13, R445-R446 (2003).

59. Morfini, G., Pigino, G., Beffert, U., Busciglio, J. \& Brady, S. T. Fast axonal transport misregulation and Alzheimer's disease. Neuromolecular Med. 2, 89-99 (2002).

60. Schaar, B. T., Kinoshita, K. \& McConnell, S. K. Doublecortin microtubule affinity is regulated by a balance of kinase and phosphatase activity at the leading edge of migrating neurons. Neuron 41, 203-213 (2004).

61. Bononi, A. et al. Protein kinases and phosphatases in the control of cell fate. Enzyme Res. 2011, 329098 (2011).

62. Manning, B. D. \& Cantley, L. C. AKT/PKB signaling: navigating downstream. Cell 129, 1261-1274 (2007).

63. Brachmann, A., Weinzierl, G., Kämper, J. \& Kahmann, R. Identification of genes in the $b W / b E$ regulatory cascade in Ustilago maydis. Mol. Microbiol. 42, 1047-1063 (2001).

64. Schuster, M. et al. Controlled and stochastic retention concentrates dynein at microtubule ends to keep endosomes on track. EMBO J. 30, 652-664 (2011).

65. Higuchi, Y., Ashwin, P., Roger, Y. \& Steinberg, G. Early endosome motility spatially organizes polysome distribution. J. Cell. Biol. 204, 343-357 (2014).

66. Raymond, C. K., Pownder, T. A. \& Sexson, S. L. General method for plasmid construction using homologous recombination. Biotechniques 26, 134-141 (1999).

67. Treitschke, S., Doehlemann, G., Schuster, M. \& Steinberg, G. The myosin motor domain of fungal chitin synthase $\mathrm{V}$ is dispensable for vesicle motility but required for virulence of the maize pathogen Ustilago maydis. Plant Cell 22, 2476-2494 (2010).

68. Wilson, R. A., Gibson, R. P., Quispe, C. F., Littlechild, J. A. \& Talbot, N. J. An NADPH-dependent genetic switch regulates plant infection by the rice blast fungus. Proc. Natl Acad. Sci. USA 107, 21902-21907 (2010).

69. Wedlich-Söldner, R., Straube, A., Friedrich, M. W. \& Steinberg, G. A balance of KIF1A-like kinesin and dynein organizes early endosomes in the fungus Ustilago maydis. EMBO J. 21, 2946-2957 (2002).

\section{Acknowledgements}

We would like to thank Drs T. Penn, M. Martin-Urdiroz, X. Yan, Mr P. Splatt and Mrs L. Yeves-Gonzalez for their technical support. In particular, we wish to highlight the excellent support provided by Mrs S. Das-Gupta. We thank Drs G. Doehlemann and S. Treitschke for their help with lectin staining and in planta microscopy. We are grateful to receive strains and plasmids from Drs N. Clark, U. Fuchs, and G. Fink and Mrs G. Dagdas. We wish to thank Professor S. Gurr for comments on the manuscript This work was supported by the Biotechnology and Biological Sciences Research Council (BB/H019774/1; BB/J009903/1).

\section{Author contributions}

G.S. developed the research plan and the experimental strategy, directed the project, analysed the data, assembled all figures and movies, and wrote the manuscript (with the exception of Methods, which were mainly written by E.B., Y.H., M.S. and S.K.) E.B. generated 15 plasmids, 24 strains and designed the $\Delta$ rab5a experiments, analysed motor motility, diaminobenzidine precipitation and morphology of $\Delta \mathrm{rab} 5 a$ and $\Delta$ hokl mutants. Y.H. generated 9 plasmids and 13 strains, partly performed and analysed the experiments of pathogenicity, effector transcription and secretion. M.S. analysed EE motility in planta, analysed dynein motility, Mcsl secretion and effector secretion in $\Delta$ hok1, investigated co-motility of Crk1 and EEs, motility and nuclear localization of other signalling components, motility of Crk1 in planta, established the analysis of

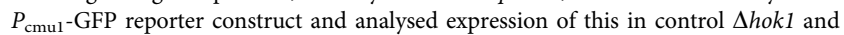
$\Delta c r k 1$ mutants. S.K. generated four strains and one plasmid, participated in the scanning electron microscopy experiments and analysed expression of effectors in $\Delta$ hok 1 mutants. N.S. established, performed and analysed plant infection assays and diaminobenzidine staining of reactive oxygen. N.J.T. contributed to writing the manuscript and participated in the research plan design. All authors corrected the manuscript and discussed the data.

\section{Additional information}

Supplementary Information accompanies this paper at http://www.nature.com/ naturecommunications

Competing financial interests: The authors declare no competing financial interests.

Reprints and permission information is available online at http://npg.nature.com/ reprintsandpermissions/

How to cite this article: Bielska, E. et al. Long-distance endosome trafficking drives fungal effector production during plant infection. Nat. Commun. 5:5097 doi: $10.1038 /$ ncomms6097 (2014)

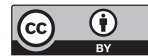

This work is licensed under a Creative Commons Attribution 4.0 International License. The images or other third party material in this article are included in the article's Creative Commons license, unless indicated otherwise in the credit line; if the material is not included under the Creative Commons license, users will need to obtain permission from the license holder to reproduce the material. To view a copy of this license, visit http://creativecommons.org/licenses/by/4.0/ 